\title{
Exercise-induced oxidative stress and melatonin supplementation: current evidence
}

\author{
Joanna Kruk ${ }^{1 *} \mathbb{D}$, Basil Hassan Aboul-Enein ${ }^{2}$ and Ewa Duchnik ${ }^{3}$
}

\begin{abstract}
Melatonin possesses the indoleamine structure and exerts antioxidant and anti-inflammatory actions and other physiological properties. Physical exercise can influence secretion of melatonin. Melatonin is used as a natural supplement among athletes to regulate sleep cycles and protect muscles against oxidative damage. Despite decades of research, there is still a lack of a comprehensive and critical review on melatonin supplementation and physical activity relationship. The aim of this literature review is to examine the antioxidant, anti-inflammatory and other biological functions played by melatonin with reference to the effect of physical exercise on melatonin secretion and the effect of this compound supplementation on exercise-induced oxidative stress in athletes. Evidence shows that intense exercises disturb antioxidant status of competitive athletes, whereas supplementation with melatonin strengthens antioxidant status in trained athletes in various sports as the compound showed high potency in reduction of the oxidative stress and inflammation markers generated during intense and prolonged exercise.
\end{abstract}

Keywords: Melatonin, Bioactivity, Oxidative stress, Inflammation, Exercise, Supplementation

\section{Background}

Melatonin (MT) $\mathrm{N}$-acetyl-5-methoxytryptamine is an endogenous indoleamine which controls crucial physiological processes such as human circadian rhythms sleep-wake cycle, anxiety, immune, and cardiac function [1-5]. MT influences appetite and regulates insulin levels among other functions [6]. The compound and its metabolites are potent antioxidants which exhibit antiinflammatory properties and protect mitochondria from damage by scavenging reactive oxygen species (ROS), and reactive nitrogen species (RNS) [7-9]. MT and its derivatives stimulate several antioxidant enzymes activity $[1,10]$. Thus, MT plays an important role in maintenance of cellular redox homeostasis and in the aging processes.

*Correspondence: joanna.kruk@usz.edu.pl

${ }^{1}$ Faculty of Physical Culture and Health, University of Szczecin, Szczecin, Poland

Full list of author information is available at the end of the article
Increase in free radicals' level and oxygen non-radical species production, followed by their accumulation in cells, may lead to a disturbance in cellular redox balance; a phenomenon called oxidative stress (OS). This state is characterized by an imbalance between pro-oxidants generation and antioxidant system's defense against ROS/ RNS and efficiency of the DNA repair system [11].

Intense physical activity (PA), similarly as other unhealthy lifestyle factors such as smoking, alcohol, improper diet, or environmental factors (e.g., radiation, viruses, and bacteria), disturbs the redox homeostasis toward oxidation $[12,13]$. Intense and prolonged exercise induces inflammation, due to high generation of free radicals and ROS/RNS and possible oxidative muscle damage $[14,15]$. In contrast, regular moderate-to-vigorous exercise generates moderate concentration of ROS/RNS, followed by adaptative responses favorable for the organism and exert beneficial effects on onset and progression of a number ROS/RNS-associated diseases [16-18]. The 
redox homeostasis in the skeletal muscle is key, in the context of sports, because the redox state depends on efficacy of ROS generation [16].

There is growing evidence that physical exercise (PE) may exert both rapid and delayed (12-24 h) effects on human MT secretion $[19,20]$.

In a previous article, we presented the enhancing effect of PE on catecholamines (CATs) secretion and on cellular redox homeostasis [21]. Evidence has shown that one of the CATs, noradrenaline (NA), is involved in the control of MT synthesis. Due to antioxidant properties MT and its metabolites, and the compound prescription in sleep disturbances of children and adolescents in neuropsychiatric disorders [22] and common ingestion by athletes, presenting the current state of knowledge on association between MT and PE is warranted. The potential of MT as an antioxidant to supplement against negative effects of exhaustive and/or high intense PE or to improve athlete's performance is necessary. Despite increasing research in this field, there is a lack of a comprehensive critical summary of the research on impact of MT supplementation to prevent against the PE-induced OS. Therefore, this article presents a representative cross-section of evidence in this area. The aim of this paper is to present the effect of PE on MT secretion and to demonstrate the current evidence on the beneficial role of the indolamine supplementation against OS generated in the human body by strenuous exercise. This review also focuses on mechanisms and determinants of exercise-induced OS and summarizes the literature data on biological functions performed by MT, especially with regard to antioxidant and anti-inflammatory activities.

\section{Chemical structure and biological activities of melatonin and its metabolites}

MT, a multifunctional and the most effective antioxidant among natural antioxidants, is endogenously produced in plants and mammals as well as supplied to the body through fruits and vegetables [1]. The compound is synthetized from tryptophan, mainly in the pineal gland in the brain (about 80\%) in darkness. Also, other sources of MT in mammals are recognized: bone marrow cells, retina, skin, platelets, lymphocyte, and the gastrointestinal tract $[2,22]$. Four enzymatic steps of MT biosynthesis have been detailed: hydroxylation, decarboxylation, acetylation, and methylation [2, 23]. In the first step, tryptophan is metabolized by tryptophan hydroxylase 1 to 5 -hydroxytryptophan. Next, the intermediate is converted by the enzyme 5-hydroxytryptophan-decarboxylase to 5-hydroxytryptamine (serotonin). Further, serotonin in mammals is metabolized to $\mathrm{N}$-acetyl-5-hydroxytryptamine by serotonin $\mathrm{N}$-acetyltransferase and methylated by the enzyme $\mathrm{N}$-acetylserotonin $\mathrm{O}$-methyltransferase to MT [23].

It is important to note that the intermediate compound in the MT biosynthesis-serotonin is a key neurotransmitter having a wide spectrum of functions in the human body [24]. For example, serotonin plays an essential role in behavior and regulation of central nervous system. MT modulates several functions, such as anxiety, psychological stress, learning, sleep, mood, pain, appetite, and exhibits antioxidant and antidepressant properties, among others. MT is carried by the systemic circulation to central and peripheral tissues (e.g., liver, pancreas, lung, kidney, heart, and the fetal adrenal gland) where functions are organized through a $24-\mathrm{h}$ cycle (so-called circadian rhythms). Moreover, the neurohormone receptors are present in several central and peripheral tissues, e.g., heart and coronary blood vessels, adrenal gland, lung, kidney, prostate, skin, $\mathrm{T}$ and $\mathrm{B}$ lymphocytes, and adipocytes.

\section{Chemical structure of melatonin and its metabolites}

Melatonin contains an electron-rich aromatic indole heterocycle with methoxy group attached to $\mathrm{C} 5$ atom and amide group attached to $\mathrm{C} 3$ atom from indole ring $[7,25$, 26] (Fig. 1).

The presence of an indole ring allows MT to function as an electron donor, thus, to reduce level of electrophilic species in cells. The site chains enable MT to exhibit both hydrophilic and lipophilic properties and diffuse across the cell membranes [27], thus, to protect biomolecules, such as cytosol, mitochondria, and nucleus against oxidation $[1,9]$. In addition, the methoxy and amide groups enhance free radicals' scavenging ability of the electronrich indole moiety, and the electronic structure of indole ensures high resonance stability and its reactivity toward ROS $[8,9,28]$. Evidence showed that $\mathrm{C}$-atoms in the indole ring structure are appropriate sites for hydroxyl radical ( $\mathrm{HO}^{\circ}$ ) and nitric oxide radical (NO) acceptation due to low energetic barrier, followed by adducts formation. Evidence simultaneously has suggested several mechanisms responsible for the MT interaction with free radicals [29] as follows: (a) an electron transfer reaction; this interaction leads to the MT cation formation which exhibits long lifetime and may react with $\mathrm{O}_{2}^{-}$; (b) hydrogen atom donation, this capacity has been ascribed to the $\mathrm{NH}$ group; (c) radical addition reaction, this process is highly efficient with powerful electron acceptors efficacy; in this way MT may deactivate two HO radicals; (d) substitution reaction, this process occurs on carbon atoms, the $\mathrm{C}$-atoms at position 2, 3 or 7 of the indole ring are easily substituted by $\mathrm{HO}$; (e) nitrosation reaction, in this process $\mathrm{N}$-nitrosomelatonin is generated as a result of the neurohormone reactivity towards NO. 


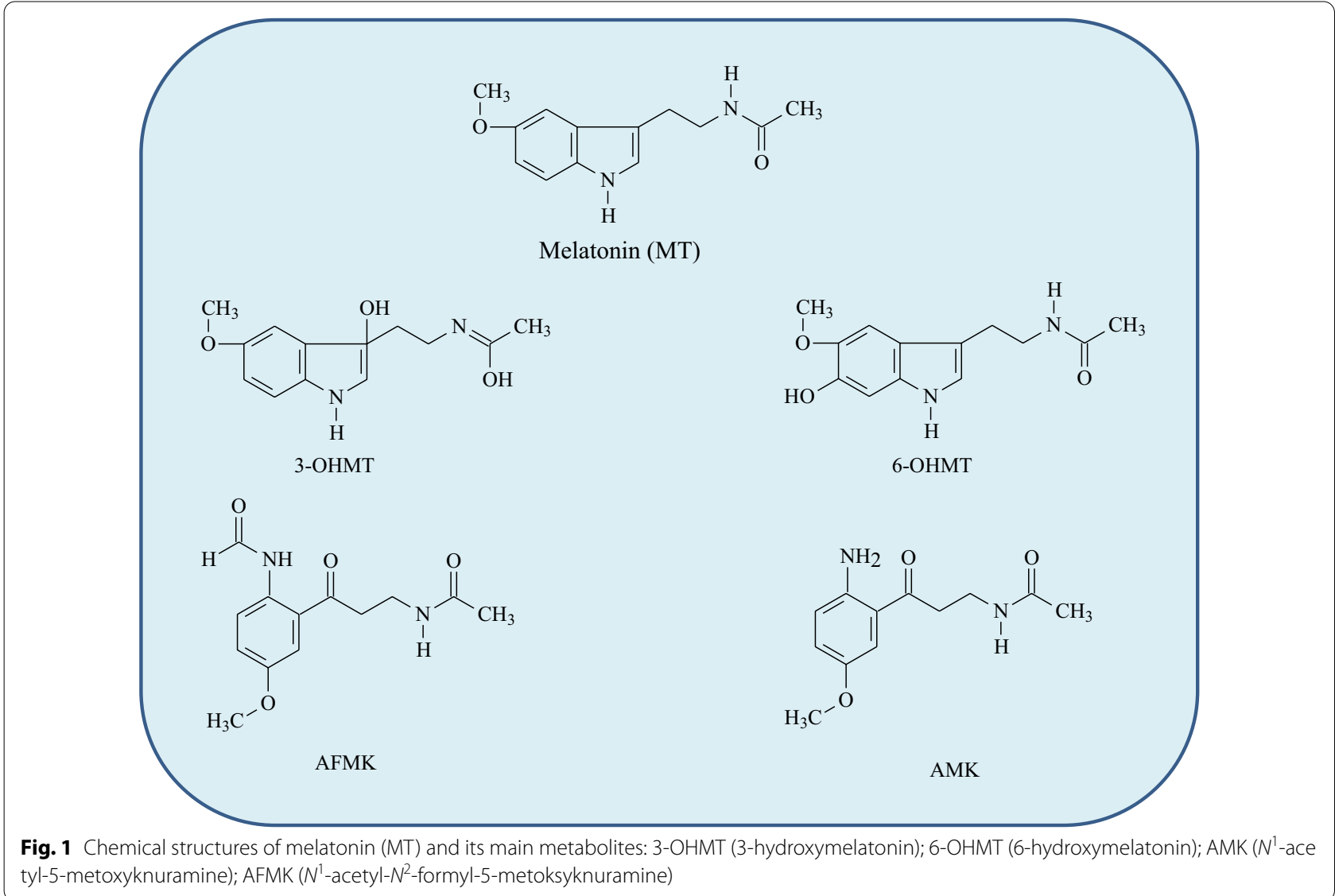

Several distinctive activities of MT have been reported due to its chemical structure. Briefly, evidence showed enhancing DNA repair by affecting genes participated in DNA damage [30,31]. These properties enable MT to scavenge peroxyl radical (ROO), singlet oxygen $\left({ }^{1} \mathrm{O}_{2}\right)$ and peroxynitrite $\left(\mathrm{ONOO}^{-}\right)$, and to inhibit the activity of $\mathrm{NO}$ synthase and hypochlorous acid $(\mathrm{HClO})$, among other toxic radicals and species $[1,32]$. The basic property that distinguishes MT from other commonly applied antioxidants is its ability to scavenge up to ten of ROS/RNS, thus MT is much more potent antioxidant than the classic antioxidants, e.g., GSH or vitamins $\mathrm{C}$ and $\mathrm{E}$ which scavenger at most one radical [25]. Another distinctive property is transformation of MT on oxidative and enzymatic pathways to several metabolites, which are widely recognized as good free radical scavengers [7, 32]. The main metabolites of MT generated during its reaction with ROS/RNS and/or on the enzymatic pathway, possessing antioxidative properties, include 3-OHMT, 6-OHMT, AMK, and AFMK as are shown in Fig. 1 [25, 28]. The MT metabolites containing hydroxyl group in their chemical structure exhibit lower potency in the scavenging ability than MT and AFMK and AMK metabolites.

\section{Biological activities of melatonin and its metabolites}

Melatonin and its metabolites exhibit a wide spectrum of both direct and indirect physiological effects in humans [4, 25, 31-34] (Fig. 2). Firstly, these compounds scavenge free radicals and other non-radicals ROS/RNS directly, reducing level of OS, thus show antioxidant abilities preventing inflammation. Secondly, these biomolecules participate in immunomodulation, improve immune defense, and exhibit other physiological activities, e.g., regulate circadian rhythms, body temperature, increase physical performance and glucose uptake in muscles, and prevent against lipid accumulation, among others [22, 35-38].

\section{Free radical scavenging and anti-inflammatory abilities of melatonin}

The main mechanism describing oxygen-based free radical scavenging by $\mathrm{MT}$ occurs via electron donation. For example, donation of an electron to $\mathrm{HO}^{\circ}$ leads to its deactivation accompanied by the MT transformation to almost non-toxic radical form (indolyl radical cation). Further reactions of indolyl radical cation with $\mathrm{HO}$ leads to the formation of several hydroxylated products (e.g., 3-OHMT, 6-OHMT) also having antioxidant 


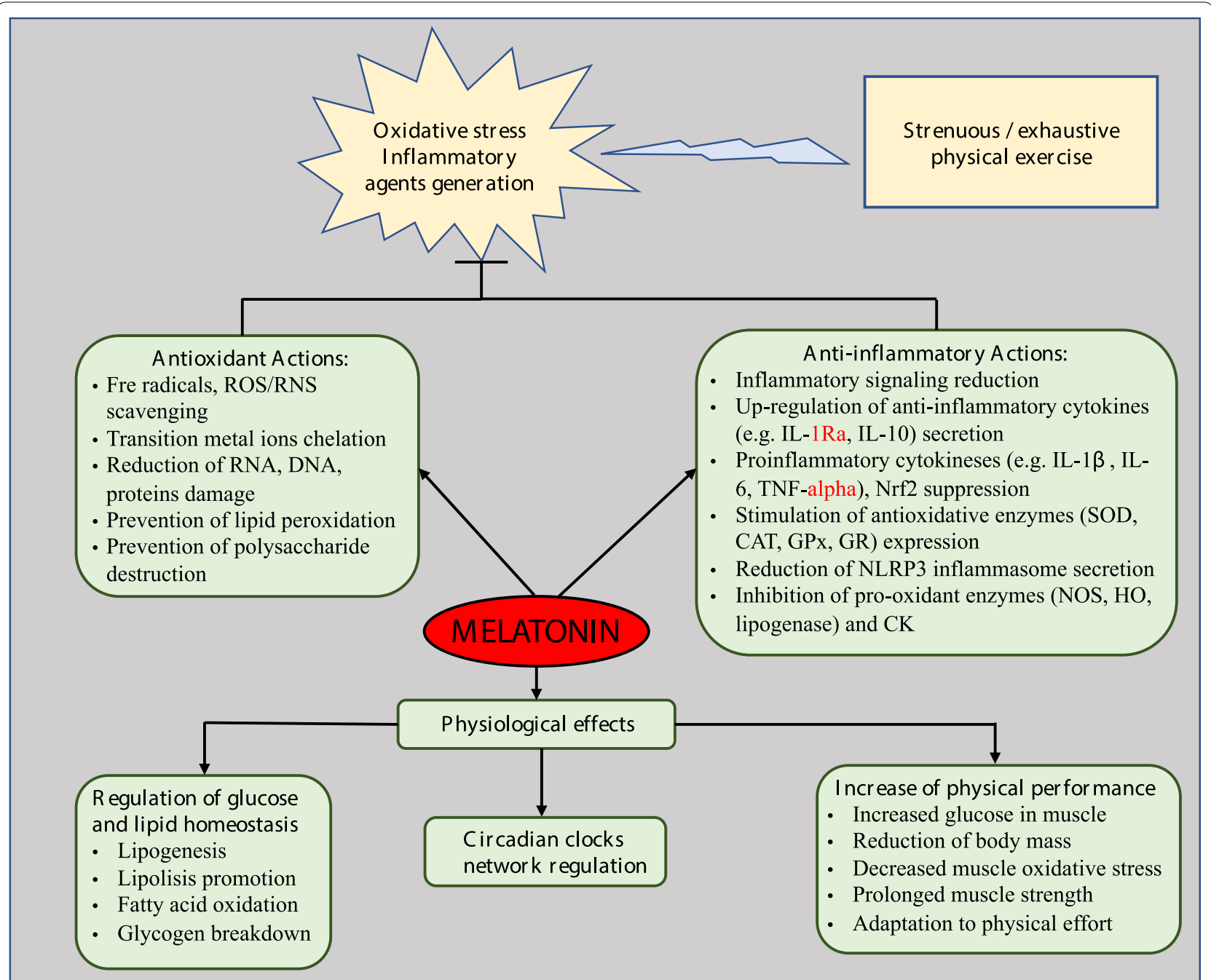

Fig. 2 Simplified scheme for the biological functions performed by melatonin

properties [7, 25, 26, 29]. Moreover, evidence shows that the AFMK and AMK metabolites (Fig. 1) react with $\mathrm{HO}^{\circ}$ in hydrophilic and lipophilic media at diffusion controlled-rates ranging from 1.2 to $7.5 \times 10^{10} \mathrm{M}^{-1} \mathrm{~s}^{-1}$ and with trichloromethylperoxyl radical $\left(\mathrm{OOCCl}_{3}\right)$, although they do not show reactivity towards hydroperoxy radical (HOO') [32]. The reactivity of MT and its derivatives exhibit continuous protection of biomolecules against OS, reducing concentration of the most dangerous to cell radicals [26, 32]. The order of the relative scavenging activity on the whole is as follows: AFMK > MT > AMK [1], although it is dependent on the environment polarity [26]. In turn, the hydroxylated metabolites of MT, such as 2-HOMT formed during MT reaction with $\mathrm{HClO}$ as well as 4-OHMT and 6-OHMT not only directly scavenge free radicals, but also through removing of transition metal ions in the redox state (e.g., $\mathrm{Fe}^{2+}, \mathrm{Cu}^{+}$) diminish concentration of
$\mathrm{HO}$. Another important antioxidant property of $\mathrm{MT}$ is decomposition of $\mathrm{H}_{2} \mathrm{O}_{2}$ and the ability to quench ${ }^{1} \mathrm{O}_{2}$ $[1,39]$.

MT and its metabolites (3-OHMT, AFMK, and AMK) also protect against metal toxicity acting as antioxidant chelating agents on two manners: via direct chelation mechanism and deprotonation reaction linked with metal ion chelation [39]. It was found that the above molecules completely inhibited OS induced by the $\mathrm{Cu}^{2+}$-ascorbate mixture, and metabolite 3-OHMT exhibited the highest efficiency [1]. The chelation activity of MT originates from the chemical structure, i.e., presence of two oxygen and two nitrogen atoms acting as di-, tri-, and tetra-dentate ligands with transition metals [40]. MT and its metabolites prevent against a free radical chain reaction of lipid peroxidation where MT and its metabolites act as inhibitors of free radicals initiating the lipid peroxidation, thus protect cells from 
the harmful effect of the peroxidation products containing a carbonyl group [41].

Further, MT and its metabolites possess ability to reduce chronic and acute inflammation [34]. Although inflammation is a natural response of the human immune system to initiate stress stimuli acute and/or chronic inflammation potentially leads to tissue damage of several organs and possibly to disease [42]. An inflammatory process results from the loss of balance between proinflammatory cytokines and anti-inflammatory agents' levels towards activation of nuclear factor $\kappa B(N F-\kappa B)$ signaling [43]. The nuclear factor penetrates the nucleus and binds to specific components modulating transcription of proinflammatory genes [42].

MT and its metabolites can exert their anti-inflammatory effect through inhibition of the transcription factors involved in proinflammatory cytokines generation, e.g., activator protein 1 (AP-1), hypoxia-inducible factor-1 $\alpha$ (HIF-1 $\alpha)$, nuclear factor erythroid 2-related factor2 (Nrf2), NF- $\mathrm{BB}$, prostaglandin synthesis, reduction of production of adhesion molecules, infiltration of neutrophils in tissue, decrease of COX-2 and inducible nitric oxide synthase (iNOS) expression as well as by increasing superoxide dismutase (SOD), CAT, glutathione peroxidase (GPx), and T-helper immune response. These actions of MT reduce inflammation and decrease further ROS formation [22, 40]. For this reason, MT is the antioxidant of great interest for medicinal purposes as OS and chronic inflammation are associated with an increased risk of several cancers [44].

Another noteworthy beneficial property of MT is its capacity to protect against cytotoxicity of heavy metals, such as lead, arsenic, chromium, cadmium, and aluminum, induced through activation of basic regulators of antioxidant inflammatory pathways: NF- $\mathrm{kB}$ and Nrf2. Evidence demonstrates their opposing actions: Nrf2 regulates antioxidant pathway via promotion of the transcription of antioxidant enzymes and cytoprotective genes, while NF-kB is the promotor of inflammatory pathways; both transcription factors are regulated by OS [45]. MT induces dissociation of Nrf2 from Keap-1 (Kelch-like ECH-associated protein), which can translocate into the nucleus, where it upregulates and promotes the transcription of antioxidant genes. In addition, Nrf2 can also bind to NF- $\mathrm{KB}$ target genes and suppress their transcription $[40,45]$. The antioxidative, anti-inflammatory abilities of MT and its metabolites as well its high lipophilicity also predispose this compound to reduce the aging process, and age-related diseases, e.g., neurological Alzheimer's disease, Parkinson's disease, or Huntington's disease, among others $[9,46]$.

\section{Anti-tumor effect of melatonin}

There is growing scientific evidence that both OS and psychological stress have been linked with alterations in the immune system responses. The disturbance manifests by a decrease in immune natural killer (NK) cells activity and their ability to respond to the proinflammatory cytokines. NK cells and tumor antigen cytotoxic $\mathrm{T}$ lymphocytes (CTLs) are important immune anticancer agents of which activity is enhanced by MT. Activation of CTLs by heat shock protein (HSP70), ATP and high mobility group box1 (HMGB1) leads to the secretion of anticancer cytokines, such as interferon gamma (IFN$\gamma)$ and TNF-alpha which can induce apoptosis in cancer cells [47]. The hormone also reduces an activity of $\mathrm{T}$ regulatory cells (Treg) and cancer-associated fibroblasts of the agents recognized as promoters of the growth and invasion of tumor cells [47]. Cancer cells show over-proliferate ability through modulation of protein expression and the signal transduction pathways such as HIF-1, NF-kBs, phosphoinositide 3-kinase/protein kinase $\mathrm{B}$ (P13K/AKT), insulin-like growth factor receptor (IGF-1R), cyclin-dependent kinases and estrogen receptor signaling [48]. MT possess the ability to selectively block the signaling pathways of tumor cells participating in metastasis by modulation of cell-cell and cell-matrix interactions and can restrain the proliferation of cancer cells and inhibit their growth [48, 49]. An important property of MT is an ability of regulation of P13K/AKT intracellular signaling pathway which is involved in the promotion of metabolism, growth, proliferation, cells survival, and angiogenesis. The P13K/AKT pathway is overactive in many cancers reducing cells apoptosis [48]. Although the precise molecular mechanisms that would allow MT to affect tumor initiation and progression are not fully understood, several other beneficial effects have been demonstrated, e.g., participation in maintaining the genomic integrity, due to its antioxidant and inflammation activities, inhibition of androgen and estrogen formation and activity and also their receptors, dysregulation of tumor metabolism [47, 48, 50]. The latter activity of MT results from its capacity to switch glucose metabolism in cancer cells from the glycolytic anaerobic category in the cytosol to a normal oxidative phosphorylation for ATP synthesis in the mitochondria and coverts pyruvate to acetyl-coenzyme A. In this way, MT can decrease energy supply needed for ATP formation and limit a rapid cancer cells growth and proliferation $[44,51$, 52]. Evidence has demonstrated that in tumor cells glycolytic anaerobic metabolism definitely overweight the oxidative metabolism; pyruvate formed in cytosol during glucose metabolism is not transformed to the mitochondria but is metabolized to lactate in cytosol [Warburg effect]. The Warburg effect limits the efficient production 
of ATP and citrate, which could arrest glycolysis as in the normal cells, and, in addition, it prefers intracellular alkaline environment, enhances glycolysis and cells cycle progression, cancer cell growth, aggressiveness, resistance to apoptosis, and makes cancer therapy difficult [51, 52]. Evidence showed that the MT effect is concentration dependent as cancer cells showed the highest cytostatic glycolysis during the day and nighttime exposure to light [51]. Findings have documented the oncostatic role of MT against several types of cancer (breast, prostate, oral, gastric, lung, brain, ovarian, colorectal, liver, renal) $[50,53]$. The compound has also been shown to be useful in cancer treatment during chemotherapy and radiotherapy $[48,54,55]$. The benefit in the MT application in cancer therapy results from its different effects on tumor cells and the normal cells. The use of MT in chemotherapy allows to reduce the dose of applied drugs to destroy tumor cells through their increased sensitivity on drugs interaction which limits their resistance [56]. In addition, MT decreases side effects of the cancer chemotherapy, increases rate of patients survival and improves overall well-being. Similarly, combination of MT with radiotherapy has also been found to restrict damage of the normal tissue related to radiation dose and achieve greater cancer inactivation; MT plays role of a radiosensitizer [54, $55]$.

\section{Other physiological function of melatonin}

Evidence documents that the primary physiological function of MT is to delivery information about the diurnal cycle of light/darkness to the human body and synchronization of central and peripheral oscillators distributed in tissues and organs [5, 57]. The circadian clock system plays a key role in maintenance of homeostasis and human health. The circadian time keeping system is composed of a generator of circadian rhythms located in suprachiasmatic nuclei (SCN) of the hypothalamus which synchronizes the peripheral cellular clocks with daily periodic environmental changes [19]. This MT action as a time indicator to the biological clocks allows humans rapidly adept themselves to environmental conditions and survive. This function is important as the circadian rhythm disturbance can increase the risk of metabolic, cardiovascular, and mental diseases, as well as cause poor quality of life $[1,2]$. The circadian rhythm of the hormone synthesis and secretion is closely related to the rhythm of sleep [58]. MT stabilizes circadian rhythms and exerts the chronobiotic effects acting on the plasma membrane trough $\mathrm{G}$ protein-dependent receptors type 1 and type 2 called MT1 and MT2, and its rhythmic release is regulated by a central circadian rhythm generator $[57,59]$. Density of MT1 and MT2 receptors in the SCN is high, although the receptors are also distributed in peripheral tissues and organs (e.g., adipose tissue, liver, heart). The signals generated by SCN induce the MT synthesis in darkness, and the process is controlled by $\mathrm{SCN}$ via the sympathetic nervous system (SNS) [57].

Importantly, MT is effective in adjusting sleep-wake cycles and improving the quality of sleep and is commonly used as a medicine for the treatment of sleep problems, e.g., in insomnia, jet leg [60]. With age, the production of MT decreases [22] which may have an influence on poor sleep quality in elderly individuals and cause the prevalence of comorbid the mental and physical health disorders. The severity of sleep impairments correlate with the decrease of endogenous MT formation and its concentration in cerebrospinal fluid [22]. It is important to note that several MT receptor agonists can also mimic MT activation, thus they are able to regulate the body sleep-wake rhythm through their actions on the MT receptors MT1 and MT2 in the SCN. Ramelteon, agomelatine and tasimelteon are representative MT receptor agonists used for sleep disorders $[58,60]$. It is maintained that the MT receptor agonist connecting to the MT1 receptor subtype can directly improve sleep latency and quality, whereas the agonist action on the MT2 receptor subtype positively influences the circadian system, e.g., by promoting early sleep. Also, the use of the MT receptors to treat comorbid insomnia in several health disorders (neurological, metabolic, cardiovascular, psychiatric) is of great importance [60-62]. The MT receptors agonists, similarly as MT, have an influence on several signaling pathways, exhibit free radical scavenging ability, stimulate the activity and/or expression of antioxidant enzymes, and suppress the proinflammatory cytokines release. For example, Wang et al. [61] reported that ramelteon administration in C57BL/6 mice cells elevated traumatic brain injury, prevented against OS and inflammation. In this study, treating with the MT agonist significantly increased the expression of IL-4, IL-10, SOD, GSH, GSH peroxidase and decreased levels of IL- $1 \beta$, TNF- $\alpha$ and MDA. The authors also observed that ramelteon decreased KEAP1 expression of a key sensor of oxidative and electrophilic stresses, promoted Nrf2 nuclear accumulation, and increased levels of heme oxygenase-1, that is Nrf2 regulated gene having antioxidant and anti-inflammatory effect in vascular cells. Ramelteon also controlled the redox controlling enzyme NQO1 (NAD $(\mathrm{P}) \mathrm{H}$ : quinone oxidoreductase 1) activity. NQO1 plays significant role in quinone metabolism and exhibit the defense against OS [63]. Further, the reduced expression of proinflammatory cytokines (IL-6, IL-1 $\beta$, TNF- $\alpha$ ), glial fibrillary acidic protein and protection against increased expressions of 
proinflammatory COX-2 and against a potent inflammatory mediator prostaglandin $\mathrm{E}_{2}\left(\mathrm{PGE}_{2}\right)$ as well as excessive $\mathrm{NO}^{\circ}$ production were reported [62].

In addition to well-established functions outlined above, MT has also been identified as an important promotor of a healthy metabolic rate through glucose homeostasis re-establishment and lipid metabolism regulation [36]. These physiologic effects of the indolamine have drawn great attention in the recent years $[4,5]$. Evidence shows MT can regulate glycogen breakdown to glucose, preserve glycogen stores, improve insulin resistance in the skeletal muscle and liver, and reduce obesity, protecting from several diseases caused by glycogen storage [6]. These MT properties influence physical performance during PE of high intensity [64]. Although evidence on the beneficial metabolic effects of MT is reported and findings showed that the hormone exhibits very low side effect [4], there is no full agreement in this respect (as reviewed by Garulet et al. [37]). Detailed discussion of the physiologic activities may be found in the reviews [4, $5,36]$.

\section{Physical exercise and melatonin}

Proper sleep and recovery are essential to athletes' benefit as well as enhancing their physical performance from training programs. Evidence has accumulated showing that athletes commonly use sleep-enhancing supplements, and MT is one of the most used aid in this respect, due also to other positive effects on the body, e.g., as an antioxidant in protection of muscles against OS and enhancer of performance [19].

\section{Influence of physical exercise on cellular redox state}

The effect of PE on the cellular redox homeostasis has been widely studied since the 1970 s, especially regarding exercise of moderate intensity $\left(50-<60 \% V \mathrm{O}_{2}^{\max }\right)$ and exercise of vigorous/high intensity $\left(\geq 60 \% V \mathrm{O}_{2}^{\max }\right)$. Independent of exercise type and intensity, exercise $\geq 50 \%$ $V \mathrm{O}_{2}^{\max }$ induces an increase of ROS/RNS concentration above physiological level, and their concentration is dependent on several factors (e.g., exercise determinants, postural position during exercise, state of training, age, gender, and diet) [65]. During exercise non-mitochondrial sources of ROS are the main contributor to overall amounts of generated species in the skeletal muscle, while during rest and recovery the mitochondrial source is decisive [66].

There is significant evidence-based research carried out during the past four decades that regular exercise of moderate intensity is a key factor regulating the level of ROS/RNS in a cell protection against chronic cell exposure to these species if their level is low $[67,68]$. ROS/ RNS are formed constantly in various tissues even at rest as a part of normal metabolic processes. They play role of secondary messengers in intracellular signaling, participate in gene expression, support cellular proliferation and relaxation of vascular smooth muscle cells, and regulate angiogenesis, among others [12, 69]. Primarily, $\mathrm{PE}$ increases mitochondrial metabolism and ROS/RNS production, followed by an increase in stress resistance and is responsible for variety of physiological adaptations (e.g., mitohormesis) [70]. Further, regular moderate-tovigorous $\mathrm{PE}$ regulates the energy expenditure, insulin-like growth factor-1 (IGF-1), and insulin-like growth factor binding proteins (IGFBPs) balance, defenses against inflammation, and enhances the immune system functioning. Exercise improves metabolism of all the human biological systems and cells as well as tissues tolerance to higher concentration of ROS generated during exercise training [71], enhancing activity of antioxidant enzymes in muscles and mitochondrial biogenesis $[9,10]$.

Besides the beneficial effect of PE on humans, evidence shows acute bouts of long-lasting and high-intensity endurance exercises can cause biochemical and hormonal disturbance, generate ROS/RNS at the rate exceeding the endogenous antioxidant system performance and disrupting redox homeostasis, i.e., induce OS in untrained subjects [72]. In addition, diet poor in antioxidants enhances the stress. Under such type of exercises the maximal consumption of molecular oxygen in the muscle fibers may reach 100-fold increase compared with the rest state. Then, skeletal muscles produce large amounts of free radicals, ROS and RNS, including $\mathrm{HO}^{\circ}$ and $\mathrm{NO}$, and are considered as the major source of these toxic species $[14,65,66]$. The increased production of the reactive species in muscles includes mainly mitochondrial nicotinamide adenine dinucleotide phosphate (NADPH) oxidase, phospholipase A2, and reactions catalyzed by xanthine oxidase $(\mathrm{XO})$, such as a breakdown of ATP accompanied by generation of adenosine diphosphate (ADP) and adenosine monophosphate (AMP) and further hypoxanthine via the adenylate kinase reaction [13, 14, 73]. Evidence summarizes possible adverse effects of acute exercise and/or overtraining, such as DNA/ RNA and protein damage, increased lipid peroxidation, decreased natural killer (NK) cells function and $\mathrm{T}$ cell activities, increased angiogenesis, induction of protooncogenes, activation of transcription factors, alternation in the muscle genome, the immune system damage, and change of signal transduction $[14,66]$. Acute reduction of the muscle action and their fatigue was described to high level of ROS produced during muscle contraction $[15,65]$.

The damaging effect of OS has been confirmed experimentally by identifying changed level of OS markers in blood and during muscle biopsies [74-76]. These 
processes may lead to chronic inflammation and increase a risk of several civilization diseases, including cancer development, progression and metastasis (more detailed reviews on this topic are available, e.g., Ref. [71]). Evidence for the adverse oxidative modifications of muscular biomolecules were mainly reported after acute or chronic anaerobic exercise [74, 77].

Acute high-intensity PE can cause biochemical and hormonal disturbance, increase OS, being positively correlated with overexpression of proinflammatory cytokines, such as interleukin-1 (IL-1), interleukin-6 (IL6), tumor necrosis factor-alpha (TNF- $\alpha$ ) and CRP [15]. Further, large changes in immune system activity and lipid and carbohydrate metabolism following exhaustive exercise can promote acute muscle inflammation and tissue damage [71]. Additionally, endurance athletes often experience poorer quality of sleep, due to high training load [78]. Intense training also decreased athletes endogenous MT level [79]. On the other hand, recent studies demonstrated that the redox signaling pathway participates in the chronic response of the skeletal muscle to endurance training $[15,79,80]$. This muscle activity includes an uptake of glucose, influences on insulin sensitivity, mitochondrial biogenesis, and initiation of antioxidant enzymes [81, 82].

\section{Effect of physical exercise on melatonin and serotonin secretion \\ Melatonin secretion}

Synthesis and secretion of MT is stimulated in darkness and occur with circadian rhythm. Evidence has indicated that the hormone concentration increases between 9:00 and 10:00 p.m. reaching a maximum value between 3.00 and 4.00 a.m. following by a decrease between 7:00 and 9:00 a.m. On average, the MT levels are estimated to be about $5 \mathrm{pg} / \mathrm{mL}$ during the day and $50-100 \mathrm{pg} / \mathrm{mL}$ at the night [83]. Synthesis and release of MT are modified by several factors (genetic factors, sex hormones, age, diet, light exposure, season, hypoxia, PE, diseases, drugs) [83-85]. These factors may potentially act as enhancers of MT secretion or its inhibitors. Briefly, increases in the MT production and its secretion were reported for a diet containing MT or the compounds supporting its synthesis, e.g., tryptophan, vitamins, and minerals that act as activators or co-factors during the hormone synthesis [85]. Further, extension of the dark phase during winter, regularly taking antidepressants and MAO inhibitors also increase the MT secretion [84]. Conversely, the presence of light, especially the nocturnal exposure to artificial light of blue cyanin color, use of $\beta 1$-adrenergic blockers and non-steroidal anti-inflammatory drugs, chronic alcohol consumption, and hypoxia have inhibitory effects. Additionally, MT release is decreased during aging and in patients with long-term chronic diseases, such as dementia, type 2 diabetes mellitus, rheumatoid arthritis, cancer [86]. Accumulated evidence presents the effect of PE on human MT secretion as exercise induces an upregulation of many cell processes and physiological functions, including the circadian clocks network synchronization $[19,20,87]$. Studies that tested the magnitude and direction of the circadian rhythm phase shift induced by $\mathrm{PE}$ showed that PE can both reduce and increase the MT concentrations, according to the rhythm phase shifting (advanced or delayed) or remain without effect. This interaction depends on the time of exercising during the day, the intensity of light, training level, and exercise determinants (intensity, duration, dose), regardless exercise type (aerobic, anaerobic, concentric, resistive) and gender [88]. Two modes of the exercise effect on human MT release have been observed rapid and delayed, depending on its duration and daily repeatability [20].

Exercising in the late evening during ascending phase of circadian MT release was accompanied by delaying the hormone secretion, or even inhibition, depending on exercise duration and intensity. In turn, when MT levels reached the maximal physiological value, even exercise of high intensity performed in early evening might enhance MT release. There is a suggestion that exercising near the offset of MT release or during the daytime (morning, afternoon) did not exert significant changes in plasma MT concentration. Further, among the circadian clock system external stressors, which can interact or cooperate with PE and modify neural activity and/or the circadian genes expression and change the clock time are: light, psychological stress, food, and PE. For this reason, it is difficult to control all factors affecting the MT release, and the data from literature are not yet consolidated. The major stimuli responsible for the stressors influence on the circadian clocks include glucocorticoids, sympathetic nerves, OS, $\mathrm{pH}$ changes, decreased oxygen levels in the local tissues, changed cytokines level, and temperature [89]. Evidence has shown ROS activate the redox signal transduction in the SCN and exhibit day-night variation controlling neuronal membrane excitability. Physical exercise alters sympathetic neural activity and increases MT release [19] caused by increased noradrenaline secretion during the sympathetic system stimulation [21].

Table 1 presents the representative data for the MT secretion following PE. The studies varied in many respects, as exercise duration (short time-from 1 to 8 days, long-lasting-from 2 to 12 months), exercise type, exercise intensity (moderate, moderate-to-vigorous, acute until exhaustion), training conditions (hypoxic, normoxic conditions, indoor training, outdoor training), sex, and measuring the timing of maximal secretion of MT as well as the measurement methods. An increase 


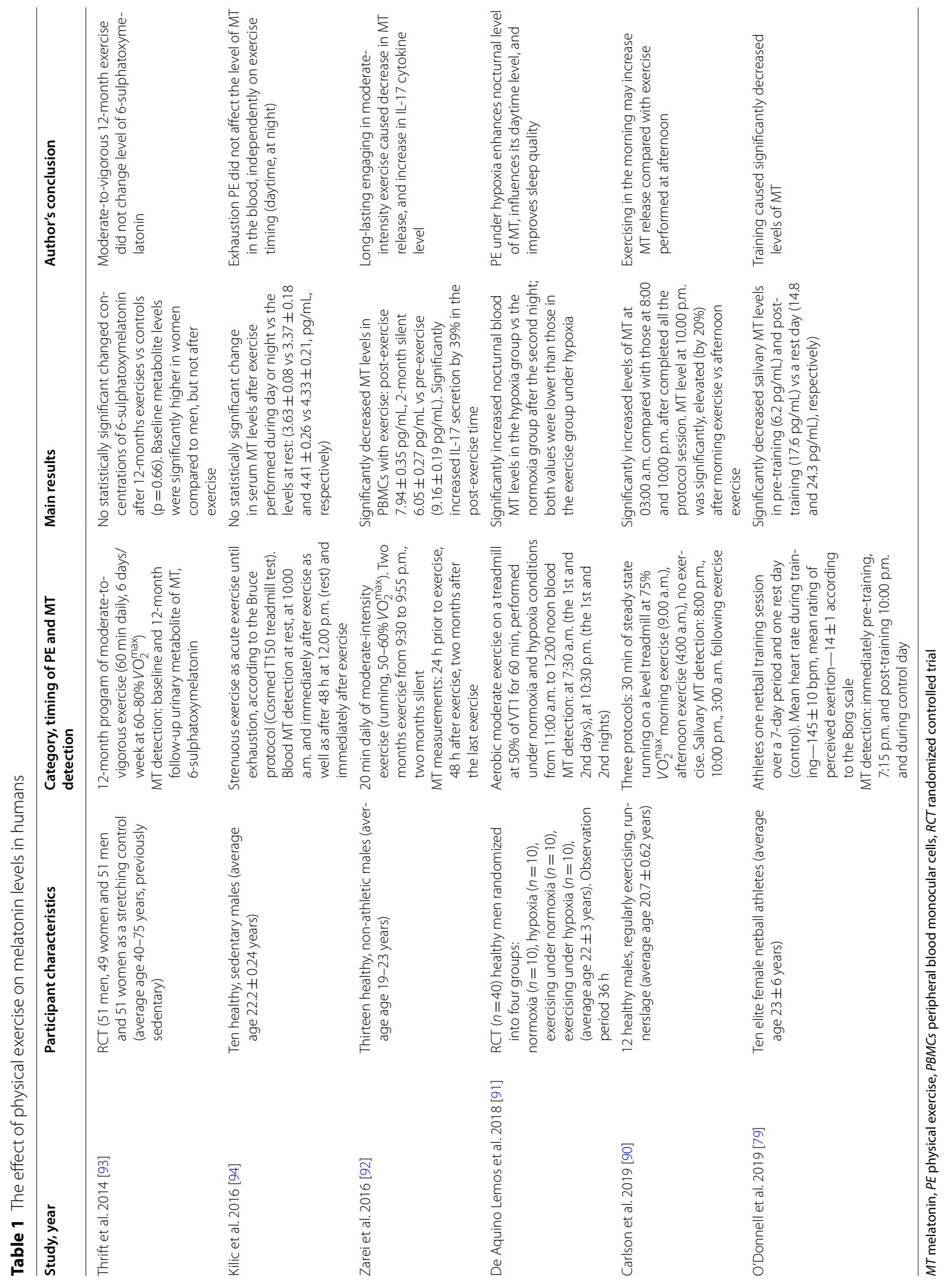


of MT concentration following PE in the morning [90] and in hypoxic conditions [91], decreases in the same $[79,92]$, and a lack of the effect $[93,94]$ were reported. Referring to the MT measurement timing, Carlson et al. [90] observed that exercise performed in the morning resulted in elevated MT secretion by $20 \%$ compared to the hormone secretion induced by the afternoon exercise. The representative studies listed in Table 1 have some limitations, which have influenced their results. These limitations include the lack of control for the major factors influencing MT secretion, the small number of measurement time points for MT detection or the small sample size.

These findings remain in accordance with previous findings because there is no clear consensus in the literature on the effect of $\mathrm{PE}$ on endogenous profile of $\mathrm{MT}$ secretion [19].

It is noteworthy to present the quantitative impact of some factors influencing the PE-MT release association. For example, earlier experimental studies of Buxton et al. reported both increases [20] and decreases [95] of MT levels following exercise depending on time of day and on intensity, duration, and type of exercise. The authors found that during exercise in nighttime, when the MT level is elevated and intensity of exercise is high, the hormone increase may reach $50 \%$. Moreover, the MT secretion was found to be dependent on individual's postural changes. These researchers maintained that moderate and high intensity exercise may influence MT levels and involve the circadian clock phase-shifting effects. Also, a study by Carr et al. [96] confirmed that plasma level of MT was transiently increased in women during all acute submaximal exercise tests of progressive endurance training after 30 -min exercise completion. A previous study by Theron et al. [97] showed that additional factors, such as BMI, lighting conditions, the time of day of exercising, and age may influence the relationship between plasma MT concentration and PE. The authors demonstrated increased plasma MT level in adult Black males who in the step-climbing exercise generated an energy output of $185 \mathrm{~W} / \mathrm{m}^{2}$ body surface area, immediately after $\mathrm{PE}$ and $1 \mathrm{~h}$ after. Evidence indicated a threefold increased MT mean level in group exercising under $320 \mathrm{~lx}$ lighting $(n=15)$ and a 2.84 -fold under 54 lx lighting $(n=15)$. Evidence exists that the season of year (winter, summer) also affects the PE-MT association, and changes of MT concentration after exercise vs before exercise may depend on the initial level of individual's endogenous MT concentration [19]. An interesting observation done by Serrano and co-workers showed professional road cyclists experienced of an adaptation to physical overloads during sports competitions [98]. It is maintained that this ability allows them to regulate intracellular OS, limiting an induction of the proinflammatory cytokines. Moreover, elevated levels of MT detected after competition, resulting from increased activity of SNS can give evidence that the protective effect of exercise operates via modulatory effect on MT secretion [57, 99]. Although processes responsible for the PE effect on MT secretion are not fully understood, several mechanisms are proposed. As maintained above, one of the biogenic catecholamines-noradrenaline participates in regulation of MT biosynthesis by the mammalian pineal gland [100]. Evidence presented high increases of noradrenaline level $\left(2 \times 10^{3} \%\right)$ in professional cyclists and even sevenfold increase after half-marathon runs [101, 102]. Exercise stimulates SNS activity and catecholamines (dopamine, noradrenaline) release [21], thus it can modulate MT secretion, followed by a net phase-shifting through the pineal gland and $\mathrm{SCN}$ of the hypothalamus, which causes an expression of receptors for MT. Further, PE stimulates the midbrain raphe nuclei activating serotoninergic input to the intergeniculate leaflets, which take part in the circadian function regulation through SCN [57]. It is suggested that an initiation of activity of the serotoninergic neurons from the median raphe nuclei is excited by serotonin and depends on its concentration in SCN [103], thus serotonin, a compound linked with well-being regulates the mammalian circadian rhythmicity.

\section{Serotonin response to exercise}

The body of evidence from animal models and human studies demonstrated the beneficial effect of exercise on brain functions $[104,105]$. Regulations of the neurotransmitters is one of several exercise's effect on brain function, among others [104]. Evidence exist that PE modulates serotonin system and can promote increased concentrations of serotonin, as muscle activation needs higher amounts of tryptophan [106]. The authors have suggested that exercise reduces levels of amino acids competing with tryptophan through muscle intake and make these amino acids easier to cross the bloodbrain barrier into muscles. In this way, serotonin and MT synthesis and their levels are increased in the brain dependently on exercise intensity. The ability of PE to modulate the activity of catecholaminergic and serotonergic systems and to enhance the brain function show that exercise as a positive activating agent of body' stress response. Additionally, aerobic exercise has a high ability to decrease depression and anxiety levels and to improve physical performance [105].

Several recent reviews have summarized experimental findings on the serotonin response to PE [104, 107, 108]. Most serotonin changes following exercise have been well documented in rodents, but only a limited number of human studies have been conducted. A review of 22 
studies in rats by Meeusen and De Meirleir presented that the majority of analyzed studies found changes in synthesis and metabolism of serotonin or its metabolites and CATs in response to exercise [107]. Another review by Basso and Suzuki [108] has analyzed research findings on the effect of exercise intensity and dose on serum serotonin in humans and rodents. The authors have suggested that acute long-term exercise increased serotonin levels and its metabolites in the frontal cortex, hippocampus, striatum, and midbrain as well as dopamine in rodents. These reviews have maintained that the serotonin increase in the central nervous system of rodents requires appropriate intensity of exercise, and the amount of serotonin released is positively correlated with PE intensity. Further, the findings of studies conducted in humans show that individuals engaged in acute exercise of low intensity may benefit from significant decreases in depression and anxiety disorders in contrast to highintensity acute exercise, which is considered to contribute to fatigue. In this regard, it is maintained that a value of exercise-induced ratio of serotonin to dopamine and noradrenaline plays a significant role in the development of fatigue and performance $[107,108]$. The authors suggested that interaction between brain serotonin and dopamine during acute PE can be responsible for the brain region specific changes in levels of serotonin.

Statistically significant increases in blood serotonin levels dependent on PE have also been reported in a few experimental studies in humans and rodents in the last decade [109-111]. A study by Valim et al. [109] showed that women with fibromyalgia $(n=22)$ randomly divided into two groups aerobic walking exercise and stretching exercise three times/week for 20 weeks experienced statistically significant increase of serum serotonin concentration in response to aerobic exercise. Stretching exercise resulted in statistically insignificant increase of serotonin level. Also, a study by Arazi et al. [110] noticed aerobic exercise significantly increased levels of serotonin and dopamine in the physically active group of 16 men addicted to opium in comparison to those who do not exercise $(n=18)$. The active group was engaged in aerobic exercise-walking 2-3 times/week for 20-30 min. The results show that even low-intensity aerobic exercise can affect the levels of these neurotransmitters. Recently these findings have been confirmed by Matsunaga et al. in rats $(n=48)$ [111]. The researchers studied the effect of intensity and timing of forced exercise (running on a motorized wheel), voluntary exercise (free running on a wheel) and low-dose exercise (voluntary, running exercise limited to $1 \mathrm{~h}$ ), through 4 weeks on levels of serotonin. They found that response of serotonin to exercise was exercise dose-dependent; significantly increased levels of serotonin and its metabolite (5-hydroxyindoleacetic acid) were seen in the dorsal and median raphe nuclei in rats engaged in forced exercise. Also, increased concentrations of the neurotransmitter were found in the paraventricular hypothalamic nucleus and caudate putamen in voluntary exercised rats. Low-dose exercise was without effect on serotonin levels, and the heavy exercised rats experienced increased anxiety-like behavior.

\section{Role of melatonin supplementation in response to exercise-induced oxidative stress}

Exogenous compounds exhibiting antiradical and antioxidant potency play a key role in regulation of ROS/ RNS concentrations. Under OS conditions, endogenous concentration of MT are unable to prevent damages to biomolecules by ROS. Evidence shows supplementation with appropriate antioxidants may improve the cellular redox homeostasis, decrease oxidative modification of DNA bases, lipids, and proteins, and decrease muscular fatigue of athletes, thus enhance exercise performance $[112,113]$. It is known that antioxidant supplementation is common practice among endurance athletes who hope to minimize OS and enhance physical performance [49, $66,114]$, and MT is a universal antioxidant that enjoys great popularity among athletes.

Table 2 lists the representative studies $(n=11)$ for the effect of MT ingestion on exercise-generated OS and inflammation.

These observational studies differ from one another mainly in terms of MT timing: taking MT before exercise [115-121] or before bedtime [122-125] and its dose, exercise type, intensity, duration and timing, and training level. The most common types of sport training in these studies are cycle ergometer, treadmill exercise or running, in which individuals mainly engaged in maximal or submaximal exercise. The authors performed measurements of OS the markers (leukocytosis, MDA, 8-OHdG, TNF- $\alpha$, IL-6, LDL, AOPP), inflammatory markers (CRP, ALAT, ASAD), TAS as well as of other endogenous antioxidants, using mainly the blood sapless. The data indicate MT intake reduced the markers of OS and inflammation in athletes engaging in severe high-intensity exercise, independently of timing of MT intake. Only three [119-121] of 11 presented experimental studies examined the MT influence on redox status followed moderate-intensity exercise. These authors found no effect of the hormone (5-6 mg doses) on biochemical and hematological parameters. This finding is in line with the evidence that at least strenuous exercise and/or overload training cause excessive production of ROS/RNS and an increase of OS [64].

As presented above, in addition to antiradical, antioxidant activities and enhancing antioxidant enzymes potency, the hormone can change glucose metabolism 
from glycolytic anaerobic category to a normal aerobic mitochondrial oxidative phosphorylation for ATP synthesis. An ingestion of MT before exercise increases use of glucose as a substrate energy. During prolonged exercise, glucose and fatty acids are utilized followed by a reduction of glycogen in muscle and liver. Evidence has shown that MT intake before PE preserves muscle glycogen stores of the compound which limits exercise performance [19].

Three of 11 presented studies [119, 120, 125] also examined effects of MT supplementation on exercise performance, finding its improvement in the two reported studies $[119,125]$. In turn, a study by Souissi et al. [121] tested the effect of MT supplementation on inflammation induced by exercise at $60 \% \mathrm{VO}_{2}^{\max }$, i.e., the lowest value of the maximum rate of $\mathrm{O}_{2}$ consumption for exercise classified as high intensity. The authors found increased levels of inflammatory markers in both the MT-supplemented group and the placebo group and no statistically significant differences between these groups.

A few studies presented in Table 2 examined also endogenous MT level in athletics after high-intensity training, observing significantly increased concentrations of inflammatory markers (TNF- $\alpha$, IL-6, IL-1Ra), isoprostane and 8-OHdG [115], malonaldehyde (MDA) [116, 118] or decreased blood levels of MT [116, 123], compared with control groups. Explaining the inconsistency of the results may originate from different training status of sportsmen, training protocols, and variability of their redox state dependently on endogenous antioxidants secretion.

It is worth noting that some studies on the effect of strenuous exercise on cellular homeostasis observed changes only in some OS markers, and even their lack $[15,126]$. Consistent with this finding, the current review by Powers et al. [10] has presented a shape of a curve illustrating the association between muscle fiber levels of ROS production induced by PE and physiological function of these species. The curve exhibits biphasic bell shape (hormesis, parabolic shape), with maximum of physiological benefit corresponding to limited muscle fatigue and exercise of moderate-to-vigorous intensity. This part of hormesis describes the normal physiological range of ROS, generated in the skeletal muscles by exercise and is considered as an important action to adapt of individuals to endurance training. In turn, the second phase of an association, described by the descending part of hormesis curve, starts from the curve maximum, and ends in the point corresponding zero of physiological functions, despite a further increase in exerciseinduced ROS generation. This part of hormesis suggests that further increase in ROS level caused by prolonged high-intensity exercise does not result in tissue damage, assuming that it is probably that PE exerts a true hormetic effect on the body [10].

In addition to MT supplementation, the level of endogenous MT was seen to be dependent on daytime performing PE and its dose. Although supplementation with MT showed high potency in reduction of OS markers formed during intense exercise, the evidence is insufficient to recommend specific dose of this antioxidant. These observations are in accordance with findings of other authors discussed earlier and below. Briefly, there is clear consensus in the literature that noradrenaline is involved in biosynthesis of MT and can determine its concentration [100]. Increased secretion of the catecholamine in plasma was observed above lactate threshold (on average of $50-80 \%$ of athletes' $\left.V \mathrm{O}_{2}^{\max }\right)[21,68]$ and was dependent not only on the level of training and duration, but also on an individual's psychological stress, health state and muscular mass engaged in training [68]. An experimental study by Kim and Kim [127], included 8 -week-old rats that were subjected to high-intensity $(n=30)$ and low-intensity $(n=30)$ exercises for $15 \mathrm{~min}$ daily over 4 weeks, demonstrated greater increase in blood MT concentration in rats exercising with highintensity exercise, compared to MT levels before and after high- and low-intensity exercise. The authors have maintained that MT secretion can be raised by exercise of high intensity, while exercise of low intensity can exert pro-oxidative effect resulting in decreased MT level due to the hormone interaction with stressors. This may explain, at least partially, the differences in findings reported in Table 2. In turn, a review by López-Flores et al., [128] of the research published in 2000-2018 (18 articles) on the effect of MT intake on sport performance underlined an important role not only a MT dose, but also daytime the supplement intake corresponding to the circadian clock system. The researchers found that ingestion of MT up to $1 \mathrm{~h}$ before PE did not improve athletic performance. The authors maintain that a dose of $10 \mathrm{mg}$ of MT consumed $30 \mathrm{~min}$ before sleep may increase the sport performance briefly. It was also suggested that supplementation with higher concentrations of this antioxidant at the time of sport training may show adverse effects, e.g., sleep deprivation and the SNS suppression. There is increasing scientific evidence, mainly based on use of vitamins $C$ and $E$, lipoic acid, and coenzyme $Q$, showing that antioxidant supplementation at high doses may be unfavorable for sport training adaptation because exogenous antioxidants can impair ROS/RNS level in skeletal muscle during sport training [129]. A previous experimental study in vitro indeed showed that MT at high concentration may also exert prooxidant activity 


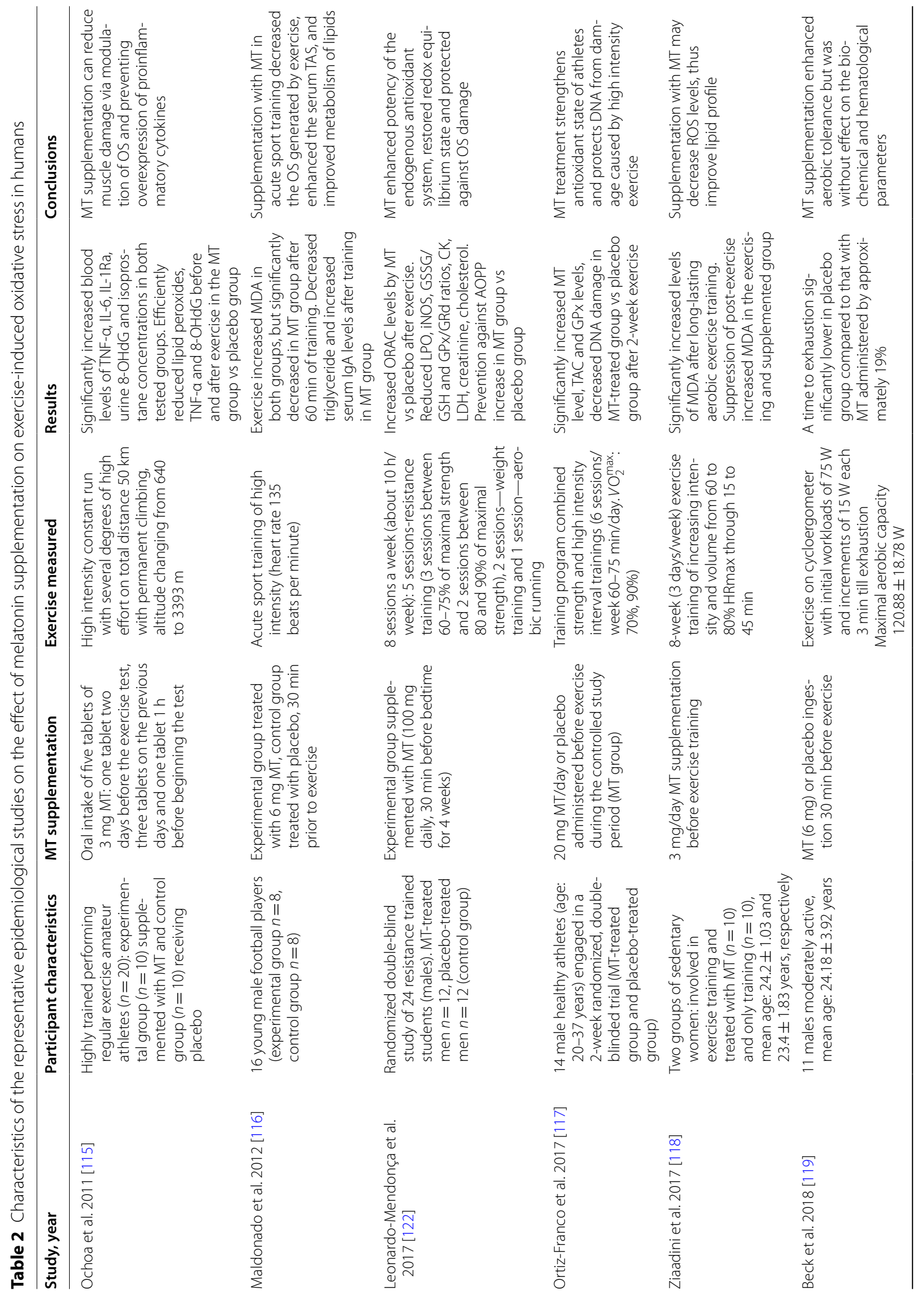




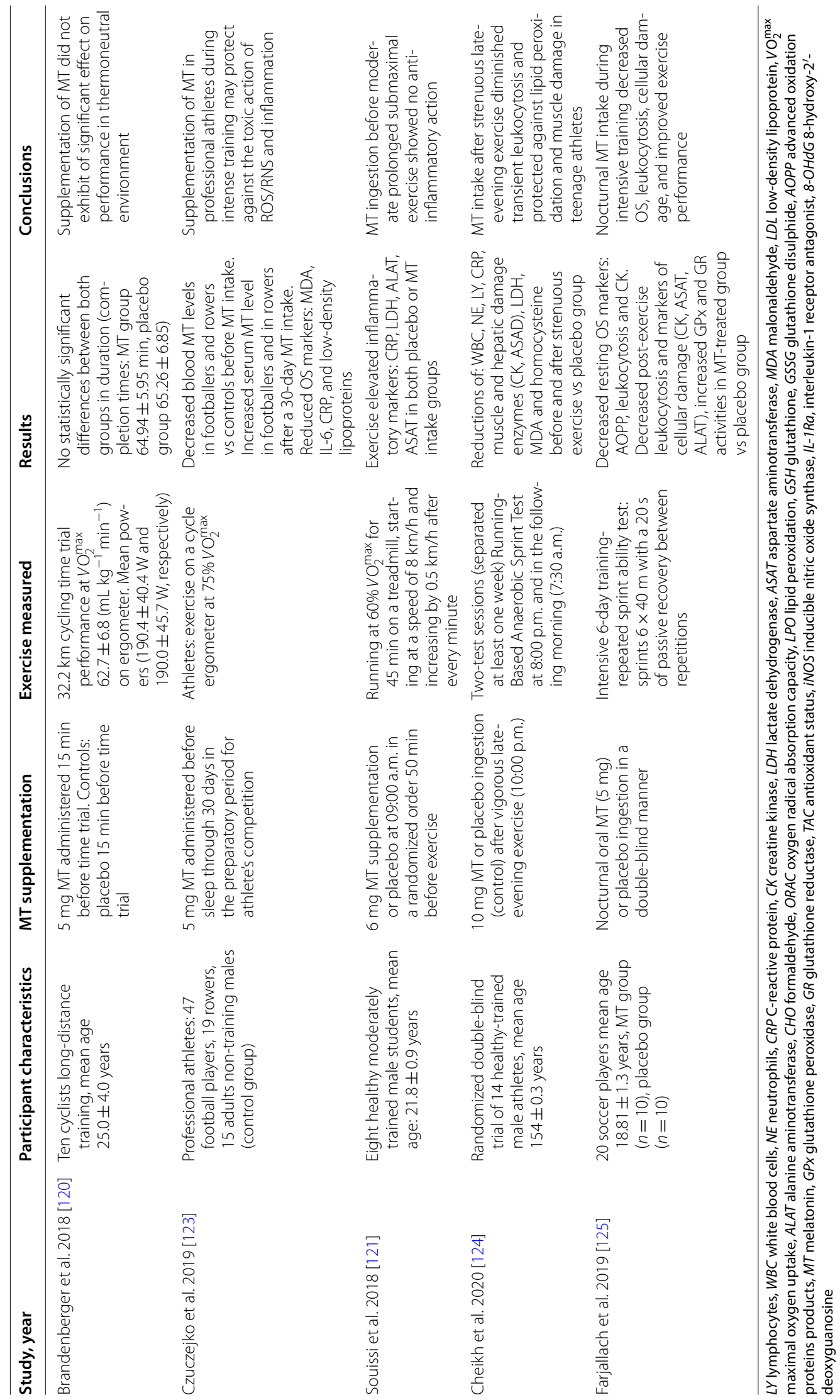


[130]. The authors observed an increase of the amount of $\mathrm{HO}, \mathrm{O}_{2}^{--}$and ${ }^{1} \mathrm{O}_{2}$ generated in the Fenton-like reaction in the presence of high concentrations of MT. Further, animal's study by Hong and colleagues reported that MT treatment with nanomolar doses of rats with the advanced knee osteoarthritis for 4 weeks alone or combined with moderate treadmill exercise $(30 \mathrm{~min} /$ day) prevented periarticular muscle damage and cartilage degeneration [131]. The authors maintain that these beneficial effects occurred through the circadian clock system, due to restoring of clock-controlled genes and correction of the abnormal chondrocyte phenotype. They observed higher reduction of serum TNF- $\alpha$ when MT intake was combined with exercise. However, prolonged MT administration to rats resulted in promotion of the proteolytic cleavage of receptor activator of nuclear factor kappa-B ligand (RANKL) protein in the synovium, followed by severe subchondral bone erosion. In turn, an animal study by Gedikli and co-workers [132] included MT injection $(10 \mathrm{mg} / \mathrm{kg})$ to Sprague-Dawley rats and studied exposition to intense exercise. The authors found cellular degenerations of kidney and liver tissues in rats engaged in exercise and a decrease in these damages in MT-treated rats. Evidence from rodents' studies documents the important regulatory and modulatory effects, supporting action of MT supplementation on animals' tissues, and skeletal muscle exposed to OS stimuli, but these findings have not yet been explored in humans. These findings show that high-dose antioxidant supplements including MT may be linked to health risk.

Another important and often discussed problem in this research area concerns adaptation to OS in sport training. There is no clear consensus in the literature that intake of antioxidants enhances adaptation to resistance exercise training. To explain the research findings incompatibility, Merry and Ristow [129] have suggested the possibility of a dose-response association between ROS/ RNS levels and exercise-training adaptation and physical performance as a hormetic response, i.e., a low dose (physiological amounts) of OS stimuli such as ROS/RNS exerts beneficial adaptive response of cells, whereas large doses of the stressors exposition, by contrast, exhibit an opposite effect (inhibition), i.e., a decrease of exercise performance. Both PE and MT can exert the beneficial effect through modulation and activation of stress resistance pathways, among others [133]. The dose-response dependence finds confirmation that both MT and physical endurance training induce antioxidant enzymes activities through increased mitochondrial content concentrations, and the Keap-1-Nrf2-ARE pathway and NF- $\mathrm{kB}$ signal transduction as a response to OS and electrophilic stress $[134,135]$.

\section{Conclusions}

This overview clearly summarizes the dispersed literature findings on MT biosynthesis, its chemical and biological properties, discusses exercise-related redox signaling and intense exercise-induced disturbances in redox homeostasis, provides the current knowledge on the effect of exercise on MT release and the effect of MT supplementation on endurance exercise-induced OS in athletes. The available literature findings confirm an important role of MT and its metabolites as a free radical and ROS/RNS scavengers, protectors of DNA damage, and reducers of the cellular and tissue oxidative damage. Current evidence highlights a wide spectrum of MT antiinflammatory and antitumor actions, OS reduction, and physiological effects on humans, such as circadian clock network regulation, glucose and lipid homeostasis regulation, and effect on physical performance. Also, utility of MT and its agonists for insomnia treatment has been demonstrated. Strong evidence suggests mitochondria are the central organelles for antioxidant actions of MT. Physical exercise alters SNS activity and MT secretion. The current evidence for the exercise dose-MT response relationship showed conflicting findings, similar to previous findings, indicating the effect of PE on the exogenous MT level in cells is extremely complex due to several factors increasing or decreasing its secretion as well as on dependencies on many factors describing exercise, an individual's redox state, training status, environmental conditions, MT intake timing, body mass, among others. $\mathrm{PE}$ increases concentration of ROS/RNS followed by activation of protein kinases, transcription factors and gene transcription, exerting beneficial adaptation or negative effects on the human body, depending on dose and exposition time of cells to this exercise. Strong experimental evidence is available for the positive effect of MT supplementation on lowering the proinflammatory cytokines, lipid peroxides, C-reactive protein, and ROS/RNS of which level could be strongly elevated in athletes engaged in prolonged endurance exercise, independently on MT supplementation timing. This compound has a potency to increase level of antioxidant enzymes and the GSH/ GSSG ratio, thus to maintain the cellular redox homeostasis in athletes engaged in acute, high-intensity sports training. Following this, epidemiological studies have found utility of MT intake to be daytime-, dose-, and exercise-dependent. The associations between PE, OS, and MT supplementation are very complex; physiological level of ROS/RNS and PE of moderate intensity are necessary to stimulate improvement of endogenous antioxidant system action.

Despite a large knowledge base on the beneficial properties of MT, the exact mechanism underlying its effect on exercise-induced disturbance in redox homeostasis 
are not yet fully understood. Also, the current research is not sufficient to indicate recommendations concerning effective but safe dose of MT intake by sportsmen. Clinical studies are needed to establish optimal MT dose, time of day and duration its supplementation to avoid adversely affecting levels of ROS/RNS signaling and consequently of the negative effect on exercise-training organism adaptation.

As the reported studies, overall, included large variations of exercise program protocols and a small sample of athletes, analyzed different markers of OS, were based on a limited extent factors increasing/decreasing the hormone release, were different in terms of MT and exercise timing and applied different MT doses, future research is needed. New research should include larger samples of athletes and address safe dose of MT intake and safe exercise training programs for different sport categories. In this sense, we hope that findings demonstrated in this review will encourage the continuation of research in this important health topic. The summarized and synthesized data of existing knowledge may help coaching staff to incorporate safe sport-specific training program, MT dose and timing. They may also be useful to research clinicians regarding the use of anti-inflammatory properties of this compound in the treatment of inflammatory diseases, including COVID-19.

\begin{abstract}
Abbreviations
'OOCCl${ }_{3}$ : Trichloromethylperoxyl radical; ${ }^{1} \mathrm{O}_{2}$ : Singlet oxygen; 3-OHMT: 3-Hydroxymelatonin; 6-OHMT: 6-Hydroxymelatonin; ADP: Adenosine diphosphate; AFMK: $N^{1}$-Acetyl- $N^{2}$-formyl-5-metoksyknuramine; AKT: Protein kinase B; ALAT: Alanine aminotransferase; AMK: $N^{1}$-Acetyl-5-metoxyknuramine; AMP: Adenosine-5'-monophosphate; AOPP: Advanced oxidation proteins products; AP1: Activator protein 1; ARE: Antioxidant responsive element; ASAT: Aspartate aminotransferase; ATP: Adenosine-5'-triphosphate; CATs: Catecholamines; CHO: Formaldehyde; CK: Creatine kinase; CRP: C-reactive protein; GPX: Glutathione peroxidase; GR: Glutathione reductase; GSH: Glutathione; GSSG: Glutathione disulphide; HIF-1a: Hypoxia-inducible factor-1a; HO: Hydroxyl radical; HOO: Hydroperoxy radical; IGF-1: Insulin-like growth factor-1; IGFBPs: Insulin-like growth factor binding proteins; IL-1: Interleukin-1; IL-1 Ra: Interleukin-1, receptor antagonist; IL-6: Interleukin-6; iNOS: Inducible nitric oxide synthase; Keap-1: Kelch-like ECH-associated protein; LDH: Lactate dehydrogenase; LDL: Low density lipoprotein; LPO: Lipid peroxidation; LY: Lymphocytes; WBC: White blood cells; MDA: Malonaldehyde; MT: Melatonin; NA: Noradrenaline; NADPH: Nicotinamide adenine dinucleotide phosphate; NE: Neutrophils; NFKB: Nuclear factor KB; NK: Natural killer; NO: Nitric oxide radical; Nrf2: Nuclear factor erythroid 2-related factor2; ONOO-: Peroxynitrite; ORAC: Oxygen radical absorption capacity; OS: Oxidative stress; P13: Phosphoinasitide-3-kinase; PA: Physical activity; PBMCs: Peripheral blood monocular cells; PE: Physical exercise; RANKL: Receptor activator of nuclear factor kappa-B ligand; RNS: Reactive nitrogen species; ROO: Peroxyl radical; ROS: Reactive oxygen species; SCN: Suprachiasmatic nucleus; SNS: Sympathetic nervous system; SOD: Superoxide dismutase; TAC: Antioxidant status; TNF-a: Tumor necrosis factor-alpha; $\mathrm{VO}_{2}$ max: Maximal oxygen uptake; $\mathrm{XO}$ : Xanthine oxidase.
\end{abstract}

Acknowledgements

None.

\section{Author's contributions}

JK, ED wrote, drafted the manuscript. BH-A edited and revised the manuscript. All authors read and approved the final manuscript.
Funding

Not applicable.

Availability of data and materials

Not applicable.

\section{Declarations}

Ethics approval and consent to participate

Not applicable.

\section{Competing interests}

The authors declare that they have no competing interests.

Consent for publication

Not applicable.

\section{Author details}

${ }^{1}$ Faculty of Physical Culture and Health, University of Szczecin, Szczecin, Poland. ${ }^{2}$ Faculty of Public Health and Policy, London School of Hygiene and Tropical Medicine, London, UK. ${ }^{3}$ Department of Aesthetic Dermatology, Pomeranian Medical University, Szczecin, Poland.

Received: 26 April 2021 Accepted: 18 August 2021

Published online: 01 September 2021

\section{References}

1. Hacisevki A, Baba B (2018) An overview of melatonin as an antioxidant molecule: a biochemical approach. Intech Open. https://doi.org/10. 5772/INTECHOPEN.79421

2. Aly HF, Rizk MZ (2018) Melatonin and its indisputable effects on the health state. Intech Open. https://doi.org/10.5772/INTECHOPEN.79817

3. Reiter RJ, Tan DX, Paredes SD, Fuentes-Broto L (2010) Beneficial effects of melatonin in cardiovascular disease. Ann Med 42(4):276-285

4. Costello RB, Lentino CV, Boyd CC, O'Connell ML, Crawford CC, Sprengel ML, Deuster PA (2014) The effectiveness of melatonin for promoting healthy sleep: a rapid evidence assessment of the literature. Nutr J 13:106

5. Rong B, Wu Q, Sun C (2020) Melatonin: a novel strategy for prevention of obesity and fat accumulation in peripheral organs through the improvements of circadian rhythms and antioxidative capacity. Melatonin Res 3(1):58-76

6. Heo JI, Yoon DW, Yu JH, Kim NH, Yoo HJ, Seo JA, Kim SG, Choi KM, Baik SH, Choi DS, Kim NH (2018) Melatonin improves insulin resistance and hepatic steatosis through attenuation of alpha-2-HS-glycoprotein. J Pineal Res 65(2):e12493

7. Tan DX, Hardeland R, Manchester LC, Galano A, Reiter RJ (2014) Cyclic-3-hydroxymelatonin (C3HOM), a potent antioxidant, scavenges free radicals and suppresses oxidative reactions. Curr Med Chem 21(13):1557-1565

8. Tan DX, Manchester LC, Qin L, Reiter RJ (2016) Melatonin: a mitochondrial targeting molecule involving mitochondrial protection and dynamics". Int J Mol Sci 17(12):2124

9. Reiter RJ, Tan DX, Rosales-Corral S, Galano A, Zhou XJ, Xu B (2018) Mitochondria: central organelles for melatonin's antioxidant and anti-aging actions. Molecules (Basel, Switzerland) 23(2):509

10. Powers SK, Deminice R, Ozdemir M, Yoshihara T, Bomkamp MP, Hyatt H (2020) Exercise-induced oxidative stress: friend or foe? J Sport Health Sci 9(5):415-425

11. Sies H (2015) Oxidative stress: a concept in redox biology and medicine. Redox Biol 4:180-183

12. Sies H, Jones DP (2020) Reactive oxygen species (ROS) as pleiotropic physiological signaling agents. Nat Rev Mol Cell Biol 21 (7):363-383

13. Kruk J, Aboul-Enein HY, Kładna A, Bowser JE (2019) Oxidative stress in biological systems and its relation with pathophysiological functions: the effect of physical activity on cellular redox homeostasis. Free Radic Res 53(5):497-521 
14. Powers SK, Nelson WB, Hudson MB (2011) Exercise-induced oxidative stress in humans: cause and consequences. Free Radic Biol Med 51(5):942-950

15. Kawamura T, Fujii R, Li X, Higashida K, Muraoka I (2018) Effects of exhaustive exercises, with different intensities, on oxidative stress markers in rat plasma and skeletal muscle. Sci Sports 33:169-175

16. Di Meo S, Napolitano G, Venditti P (2019) Mediators of physical activity protection against ROS-linked skeletal muscle damage. Int J Mol Sci 20(12):3024

17. Pedersen BK, Saltin B (2015) Exercise as medicine-evidence for prescribing exercise as therapy in 26 different chronic diseases. Scand J Med Sci Sports 25(Suppl 3):1-72

18. Gondim OS, de Camargo VT, Gutierrez FA, Martins PF, Passos ME, Momesso CM, Santos VC, Garjao R, Pithon-Curi TC, Cury-Boaventura MF (2015) Benefits of regular exercise on inflammatory and cardiovascular risk markers in normal weight, overweight and obese adults. PLOS ONE 10(10):e0140596

19. Escames G, Ozturk G, Baño-Otálora B, Pozo MJ, Madrid JA, Reiter RJ, Serrano E, Concepción M, Acuña-Castoviejo D (2012) Exercise and melatonin in humans: reciprocal benefits. J Pineal Res 52(1):1-11

20. Buxton OM, Lee CW, L'Hermite-Baleriaux M, Turek FW, Van Cauter E (2003) Exercise elicits phase shifts and acute alterations of melatonin that vary with circadian phase. Am J Physiol Regul Integr Comp Physiol 284(3):R714-R724

21. Kruk J, Kotarska K, Aboul-Enein BH (2020) Physical exercise and catecholamines response: benefits and health risk: possible mechanisms. Free Radic Res 54(2-3):105-125

22. Tordjman S, Chokron S, Delorme R, Charrier A, Bellissant E, Jaafari N, Fougerou C (2017) Melatonin: pharmacology, functions and therapeutic benefits. Curr Neuropharmacol 15(3):434-443

23. Zhao D, Yu Y, Shen Y, Liu Q, Zhao Z, Sharma R, Reiter RJ (2019) Melatonin synthesis and function: evolutionary history in animals and plants. Front Endocrinol (Lausanne) 10:249

24. Azouzi S, Santuz H, Morandat S, Pereira C, Côté F, Hermine O, El Kirat K, Colin Y, Le Van KC, Etchebest C, Amireault P (2017) Antioxidant and membrane binding properties of serotonin protect lipids from oxidation. Biophys J 112(9):1863-1873

25. Tan DX, Manchester LC, Terron MP, Flores LJ, Reiter RJ (2007) One molecule, many derivatives: a never-ending interaction of melatonin with reactive oxygen and nitrogen species? J Pineal Res 42(1):28-42

26. Reina M, Martínez A (2018) A new free radical scavenging cascade involving melatonin and three of its metabolites (3OHM, AFMK and AMK). Comput Theor Chem 1123:111-118

27. Kratz EM, Piwowar A (2017) Melatonin, advanced oxidation protein products and total antioxidant capacity as seminal parameters of prooxidant-antioxidant balance and their connection with expression of metalloproteinases in context of male fertility. J Physiol Pharmacol 68(5):659-668

28. Reiter RJ, Mayo JC, Tan DX, Sainz RM, Almatorre-Jimenez M, Qin L (2016) Melatonin as an antioxidant: under promises but over delivers. J Pineal Res 61(3):253-278

29. Tan DX, Reiter RJ, Manchester LC, Yan MT, El-Sawi M, Sainz RM, Mayo JC, Kohen R, Allegra M, Hardeland R (2002) Chemical and physical properties and potential mechanisms: melatonin as a broad spectrum antioxidant and free radical scavenger. Curr Top Med Chem 2(2):181-197

30. Liu R, Fu A, Hoffman AE, Zheng T, Zhu Y (2013) Melatonin enhances DNA repair capacity possibly by affecting genes involved in DNA damage responsive pathways. BMC Cell Biol 14:1. https://doi.org/10. 1186/1471-2121-14-1

31. Galano A, Tan DX, Reiter RJ (2018) Melatonin: a versatile protector against oxidative DNA damage. Molecules 23(3):530

32. Galano A, Tan DX, Reiter RJ (2013) On the free radical scavenging activities of melatonin's metabolites. AFMK and AMK J Pineal Res 54(3):245-257

33. Mansouri M, Abbasian S, Khazaie M (2018) Melatonin and exercise: their effects on malondialdehyde and lipid peroxidation. Intech Open. https://doi.org/10.5772/INTECHOPEN.79561

34. Favero G, Franceschetti L, Bonomini F, Rodella LF, Rezzani R (2017) Melatonin as an anti-inflammatory agent modulating inflammasome activation. Int J Endocrinol 2017:1835195
35. Ekmekcioglu C (2006) Melatonin receptors in humans: biological role and clinical relevance. Biomed Pharmacother 60(3):97-108

36. Owino S, Buonfiglio D, Tchio C, Tosini G (2019) Melatonin signaling a key regulator of glucose homeostasis and energy metabolism. Front Endocrinol 10:488. https://doi.org/10.3389/fendo.2019.00488

37. Garaulet M, Qian J, Florez JC, Arendt J, Saxena R, Scheer F (2020) Melatonin effects on glucose metabolism: time to unlock the controversy. Trends Endocrinol Metab 31(3):192-204

38. Jin JX, Lee S, Taweechaipaisankul A, Kim GA, Lee BC (2018) Melatonin regulates lipid metabolism in porcine oocytes. J Pineal Res. 62(2). https://doi.org/10.1111/jpi.12388

39. Galano A, Medina ME, Tan DX, Reiter RJ (2015) Melatonin and its metabolites as copper chelating agents and their role in inhibiting oxidative stress: a physicochemical analysis. J Pineal Res 58(1):107-116

40. Rana S (2018) Protection of metal toxicity by melatonin-recent advances. EC Pharmacol Toxicol 9(2018):851-864

41. Sagan D, Stepniak J, Gesing A, Lewinski A, Karbownik-Lewinska M (2017) Melatonin reverses the enhanced oxidative damage to membrane lipids and improves skin biophysical characteristics in formersmokers-a study in postmenopausal women. Ann Agric Environ Med 24(4):659-666

42. Chen L, Deng H, Cui H, Fang J, Zuo Z, Deng J, Li Y, Wang X, Zhao L (2017) Inflammatory responses and inflammation-associated diseases in organs. Oncotarget 9(6):7204-7218

43. Siomek A (2012) NF-kB signaling pathway and free radical impact. Acta Biochim Pol 59(3):323-331

44. Reiter RJ, Sharma R, Ma Q Rorsales-Corral S, de Almeida Chuffa LG (2020) Melatonin inhibits Warburg-dependent cancer by redirecting glucose oxidation to the mitochondria: a mechanistic hypothesis. Cell Mol Life Sci 77(13):2527-2542

45. Liddell JR (2017) Interplay between Nrf2 and NF-kB in neuroinflammatory diseases. J Clin Cell Immunol 8:1-5

46. Esposito $E_{1}$ Cuzzocrea S (2010) Antiinflammatory activity of melatonin in central nervous system. Curr Neuropharmacol 8(3):228-242

47. Mortezaee K, Potes Y, Mirtavoos-Mahyari H, Motevaseli E, Shabeeb D, Musa AE, Najafi M, Farhood B (2019) Boosting immune system against cancer by melatonin: a mechanistic viewpoint. Life Sci 1(238):116960

48. Talib WH, Alsayed AR, Abuawad A, Daoud S, Mahmod A (2021) Melatonin in cancer treatment: current knowledge and future opportunities. Molecules 26(9):2506

49. Lu KH, Lin CW, Hsieh YH, Su SC, Reiter RJ, Yang SF (2020) New insights into antimetastatic signaling pathways of melatonin in skeletomuscular sarcoma of childhood and adolescence. Cancer Metastasis Rev 39(1):303-320

50. Menéndez-Menéndez J, Martínez-Campa C (2018) Melatonin: an anti-tumor agent in hormone-dependent cancers. Int J Endocrinol 2(2018):3271948

51. Icard P, Shulman S, Farhat D, Steyaert JM, Alifano M, Lincet H (2018) How the Warburg effect supports aggressiveness and drug resistance of cancer cells? Drug Resist Updat 38:1-11

52. Zois CE, Harris AL (2016) Glycogen metabolism has a key role in the cancer microenvironment and provides new targets for cancer therapy. J Mol Med (Berl) 94(2):137-154

53. Chuffa L, Reiter RJ, Lupi LA (2017) Melatonin as a promising agent to treat ovarian cancer: molecular mechanisms. Carcinogenesis 38(10):945-952

54. Alonso-González C, González A, Menéndez-Menéndez J, MartínezCampa C, Cos S (2020) Melatonin as a radio-sensitizer in cancer. Biomedicines 8(8):247

55. Farhood B, Goradel NH, Mortezaee K, Khanlarkhani N, Salehi E, Nashtae MS, Mirtavoos-Mahyari H, Motevaseli E, Shabeeb D, Musa AE, Najafi M (2019) Melatonin as an adjuvant in radiotherapy for radioprotection and radiosensitization. Clin Transl Oncol 21(3):268-279

56. Najafi M, Salehi E, Farhood B, Nashtaei MS, Hashemi Goradel N, Khanlarkhani N, Namjoo Z, Mortezaee K (2019) Adjuvant chemotherapy with melatonin for targeting human cancers: a review. J Cell Physiol 234(3):2356-2372

57. Khullar A (2012) The role of melatonin in the circadian rhythm sleepwake cycle: a review of endogenous and exogenous melatonin. Psychiatr Times 29(7):26 
58. Satyanarayanan SK, Shih YH, Chien YC, Huang SY, Gałecki P, Kasper S, Chang JP, Su KP (2018) Anti-oxidative effects of melatonin receptor agonist and omega-3 polyunsaturated fatty acids in neuronal sh-sy5y cells: deciphering synergic effects on anti-depressant mechanisms. Mol Neurobiol 55(9):7271-7284

59. Slominski RM, Reiter RJ, Schlabritz-Loutsevitch N, Ostrom RS, Slominski A (2012) Melatonin membrane receptors in peripheral tissues: distribution and functions. Mol Cell Endocrinol 351(2):152-166

60. Laudon M, Frydman-Marom A (2014) Therapeutic effects of melatonin receptor agonists on sleep and comorbid disorders. Int J Mol Sci 15(9):15924-15950

61. Wang J, Jiang C, Zhang K, Lan X, Chen X, Zang W, Wang Z, Guan F, Zhu C, Yang X, Lu H, Wang J (2019) Melatonin receptor activation provides cerebral protection after traumatic brain injury by mitigating oxidative stress and inflammation via the Nrf2 signaling pathway. Free Radic Biol Med 131:345-355

62. Yang S, Wang J, Wang D, Guo L, Yu D (2021) Melatonin receptor agonist ramelteon suppresses Ips-induced neuroinflammation in astrocytes. ACS Chem Neurosci 12(9):1498-1505

63. Ross D, Siegel D (2021) The diverse functionality of NQO1 and its roles in redox control. Redox Biol 41:101950

64. Trionfante CP, Davis GR, Farney TM, Miskowiec RW, Nelson AG (2017) A pre-exercise dose of melatonin can alter substrate use during exercise. Int J Exerc Sci 10(7):1029-1037

65. Djordjevic D, Cubrilo D, Macura M, Barudzic N, Djuric D, Jakovljevic V (2011) The influence of training status on oxidative stress in young male handball players". Mol Cell Biochem 351(1-2):251-259

66. Mason SA, Trewin AJ, Parker L, Wadley GD (2020) Antioxidant supplements and endurance exercise: current evidence and mechanistic insights. Redox Biol 35:101471

67. de Lemos ET, Oliveira J, Pinheiro JP, Reis F (2012) Regular physical exercise as a strategy to improve antioxidant and anti-inflammatory status: benefits in type 2 diabetes mellitus. Oxid Med Cell Longev 2012:741545

68. Taheri M, Irandoust K (2018) The exercise-induced weight loss improves self-reported quality of sleep in obese elderly women with sleep disorders. Sleep Hypn 20(1):54-59

69. Meo SD, Reed TT, Venditti P, Victor VM (2016) Role of ROS and RNS sources in physiological and pathological conditions. Oxid Med Cell Longev 2016:1245049

70. Merry TL, Ristow M (2016) Mitohormesis in exercise training. Free Radic Biol Med 98:123-130

71. Magherini F, Fiaschi T, Marzocchini R, Mannelli M, Gamberi T, Modesti PA, Modesti A (2019) Oxidative stress in exercise training: the involvement of inflammation and peripheral signals. Free Radic Res 53(11-12):1155-1165

72. Neves P, Tenório T, Lins TA, Muniz MTC, Pithon-Curi TC, Botero JP, Do Prado WL (2015) Acute effects of high- and low-intensity exercise bouts on leukocyte counts. J Exerc Sci Fit 13(1):24-28

73. Burke JE, Dennis EA (2009) Phospholipase A2 structure/function, mechanism, and signaling. J Lipid Res 50(Suppl):S237-S242

74. Cuyul-Vásquez I, Berríos-Contreras L, Soto-Fuentes S, Hunter-Echeverría K, Marzuca-Nassr GN (2020) Effects of resistance exercise training on redox homeostasis in older adults. A systematic review and metaanalysis. Exp Gerontol 138:111012

75. Ammar A, Trabelsi K, Boukhris O, Glenn JM, Bott N, Masmoudi L, Hakim A, Chtourou H, Driss T, Hoekelmann A, Abed KE (2020) Effects of aerobic-, anaerobic- and combined-based exercises on plasma oxidative stress biomarkers in healthy untrained young adults. Int J Environ Res Public Health 17(7):2601

76. Kawamura T, Muraoka I (2018) Exercise-induced oxidative stress and the effects of antioxidant intake from a physiological viewpoint. Antioxidants (Basel, Switzerland) 7(9):119

77. Cubrilo D, Djordjevic D, Zivkovic V, Djuric D, Blagojevic D, Spasic M, Jakovljevic V (2011) Oxidative stress and nitrite dynamics under maximal load in elite athletes: relation to sport type. Mol Cell Biochem 355:273-279

78. Driller MW, Dixon ZT, Clark MI (2017) Accelerometer-based sleep behavior and activity levels in student athletes in comparison to student non-athletes. Sport Sci Health 13:411-418
79. O'Donnell SL, Beaven CM, Jacobson MG, Bird S, Driller MW (2019) Melatonin and sleep responses following exercise in elite female athletes. J Sport Exerc Sci 3(2):8-13

80. Mason SA, Morrison D, McConell GK, Wadley GD (2016) Muscle redox signaling pathways in exercise. Role of antioxidants. Free Radic Biol Med 98:29-45

81. Hearris MA, Hammond KM, Fell JM, Morton JP (2018) Regulation of muscle glycogen metabolism during exercise: implications for endurance performance and training adaptations. Nutrients 10(3):298

82. Knuiman P, Hopman MT, Mensink M (2015) Glycogen availability and skeletal muscle adaptations with endurance and resistance exercise. Nutr Metab 12(59). https://doi.org/10.1186/s12986-015-0055-9.

83. Wieczorek J, Blazejczyk K, Morita T (2016) Changes in melatonin secretion in tourists after rapid movement to another lighting zone without transition of time zone. Chronobiol Int 33(2):220-233

84. Rzepka-Migut B, Paprocka J (2020) Melatonin-measurement methods and the factors modifying the results. A systematic review of the literature. Int J Environ Res Public Health 17(6):1916

85. Peuhkuri K, Sihvola N, Korpela R (2012) Dietary factors and fluctuating levels of melatonin. Food Nutr Res. 56. https://doi.org/10.3402/fnr.v56i0. 17252.

86. Hardeland R (2012) Neurobiology, pathophysiology, and treatment of melatonin deficiency and dysfunction. ScientificWorld Journal 2012:640389

87. Wolff CA, Esser KA (2019) Exercise timing and circadian rhythms. Curr Opin Physiol 10:64-69

88. Zouhal H, Jacob C, Delamarche P, Gratas-Delamarche A (2008) Catecholamines and the effects of exercise, training and gender. Sports Med 38(5):401-423

89. Tahara Y, Shibata S (2018) Entrainment of the mouse circadian clock: effects of stress, exercise, and nutrition. Free Radic Biol Med 119:129-138

90. Carlson LA, Pobocik KM, Lawrence MA, Brazeau DA, Koch AJ (2019) Influence of exercise time of day on salivary melatonin responses. Int J Sports Physiol Perform 14(3):351-353

91. De Aquino LV, Dos Santos RVT, Antunes HKM, Behn C, Viscor G, Lira FS, Bittar IGL, Caris AV, Tufik S, De Mello MT (2018) Melatonin and sleep responses to normobaric hypoxia and aerobic physical exercise: a randomized controlled trial. Physiol Behav 196:95-103

92. Zarei M, Farokhi Fard G, Salehi I, Sarihi A, Zamani A (2016) Influence of physical exercise on interleukin-17, cortisol and melatonin levels in serum, whole blood and mitogen activated peripheral blood mononuclear cells. Int J Med Res Health Sci 5:77-83

93. Thrift AP, Xiao L, Patel SR, Tworoger SS, McTiernan A, Duggan C (2014) Effects of physical activity on melatonin levels in previously sedentary men and women. Cancer Epidemiol Biomarkers Prev 23(8):1696-1699

94. Kilic M, Demirhan B, Patlar S, Baltacı A, Mogulkoc R (2016) Effects of diurnal and nocturnal strenuous exercise on serum melatonin levels. Rev Bras Med Esporte 22:436-438

95. Buxton OM, Frank SA, L'Hermite-Balériaux M, Leproult R, Turek FW, Van Cauter $E$ (1997) Roles of intensity and duration of nocturnal exercise in causing phase delays of human circadian rhythms. Am J Physiol 273(3):536-542

96. Carr DB, Reppert SM, Bullen B, Skrinar G, Beitins I, Arnold M, Rosenblatt M, Martin JB, McArthur JW (1981) Plasma melatonin increases during exercise in women. J Clin Endocrinol Metabol 53(1):224-225

97. Theron JJ, Oosthuizen JM, Rautenbach MM (1984) Effect of physical exercise on plasma melatonin levels in normal volunteers. S Afr Med J 66(22):838-841

98. Serrano E, Venegas C, Escames G, Sánchez-Muñoz C, Zabala M, Puertas A, de Haro T, Gutierrez A, Castillo M, Acuna-Castroviejo D (2010) Antioxidant defence and inflammatory response in professional road cyclists during a 4-day competition. J Sports Sci 28(10):1047-1056

99. Jauhari A, Baranov SV, Suofu Y, Kim J, Singh T, Yablonska S, Li F, Wang X, Oberly P, Minnigh MB, Poloyac SM, Carlisle DL, Friedlander RM (2020) Melatonin inhibits cytosolic mitochondrial DNA-induced neuroinflammatory signaling in accelerated aging and neurodegeneration. J Clin Invest 130(6):3124-3136

100. Brownstein M, Axelrod J (1974) Pineal gland: 24-hour rhythm in norepinephrine turnover. Science 184(4133):163-165 
101. Messan F, Tito A, Gouthon P, Nouatin KB, Nigan IB, Blagbo AS, Lounana $J$, Medelli J (2017) Comparison of catecholamine values before and after exercise-induced bronchospasm in professional cyclists. Tanaffos 16(2):136-143

102. Danese E, Tarperi C, Salvagno GL, Guzzo A, Sanchis-Gomar F, Festa L, Bertinato L, Montagnana M, Schena F, Lippi G (2018) Sympathoadrenergic activation by endurance exercise: Effect on metanephrines spillover and its role in predicting athlete's performance. Oncotarget 9(21):15650-15657

103. Challet $E$ (2007) Minireview: entrainment of the suprachiasmatic clockwork in diurnal and nocturnal mammals. Endocrinology 148(12):5648-5655

104. Lin TW, Kuo YM (2013) Exercise benefits brain function: the monoamine connection. Brain Sci 3(1):39-53

105. Heijnen S, Hommel B, Kibele A, Colzato LS (2016) Neuromodulation of aerobic exercise - a review. Front Psychol 6:1890

106. Patrick RP, Ames BN (2015) Vitamin D and the omega-3 fatty acids control serotonin synthesis and action, part 2: relevance for ADHD, bipolar disorder, schizophrenia, and impulsive behavior. FASEB J 29(6):2207-2222

107. Meeusen R, De Meirleir K (1995) Exercise and brain neurotransmission. Sports Med 20(3):160-188

108. Basso JC, Suzuki WA (2017) The effects of acute exercise on mood, cognition, neurophysiology, and neurochemical pathways: a review. Brain Plast 2(2):127-152

109. Valim V, Natour J, Xiao Y, Pereira AF, Lopes BB, Pollak DF, Zandonade E, Russell IJ (2013) Effects of physical exercise on serum levels of serotonin and its metabolite in fibromyalgia: a randomized pilot study. Rev Bras Reumatol 53(6):538-541

110. Arazi H, Mollazadeh R, Dadvand SS, Davaran M (2016) The blood levels of serotonin and dopamine and physical fitness factors in active and inactive men addicted to opium during rehabilitation. Phys Activity Rev 4:1-8

111. Matsunaga D, Nakagawa $H$, Ishiwata $T$ (2021) Difference in the brain serotonin and its metabolite level and anxiety-like behavior between forced and voluntary exercise conditions in rats. Neurosci Lett 744:135556

112. Jäger R, Purpura M, Kerksick CM (2019) Eight weeks of a high dose of curcumin supplementation may attenuate performance decrements following muscle-damaging exercise. Nutrients 11(7):1692

113. Morrison D, Hughes J, Della Gatta PA (2015) Vitamin C and E supplementation prevents some of the cellular adaptations to endurancetraining in humans. Free Radic Biol Med 89:852-862

114. De León FLG, Rodriguez-Villalobos JM, Candia-Luján R, Carrasco-Legleu CE, Enriquez CLA (2019) Effectiveness of antioxidant supplements in the improvement of athletic physical performance: review article. Rev Habanera Cienc Médi 18(2):194-216

115. Ochoa JJ, Díaz-Castro J, Kajarabille N (2011) Melatonin supplementation ameliorates oxidative stress and inflammatory signaling induced by strenuous exercise in adult human males. J Pineal Res 51(4):373-380

116. Maldonado MD, Manfredi M, Ribas-Serna J, Garcia-Moreno H, Calvo JR (2012) Melatonin administrated immediately before an intense exercise reverses oxidative stress, improves immunological defenses and lipid metabolism in football players. Physiol Behav 105(5):1099-1103

117. Ortiz-Franco M, Planells E, Quintero B et al (2017) Effect of melatonin supplementation on antioxidant status and DNA damage in high intensity trained athletes. Int J Sports Med 38(14):1117-1125

118. Ziaadini F, Aminae M, Rastegar M, Abbasian S, Memari AH (2017) Melatonin supplementation decreases aerobic exercise training inducedlipid peroxidation and malondialdehyde in sedentary young women. Polish J Food Nutrn Sci 67:225-232

119. Beck W, Messias LH, Silva FC, Manchado-Gobatto F, Gobatto C (2018) Acute melatonin administration enhances aerobic tolerance: an analysis of biochemical and hematological parameters. Motriz Rev Educ Fis 24(2):e1018169
120. Brandenberger KJ, Ingalls CP, Rupp JC, Doyle JA (2018) Consumption of a 5-mg melatonin supplement does not affect $32.2-\mathrm{km}$ cycling time trial performance. J Strength Cond Res 32(10):2872-2877

121. Souissi A, Souissi N, Dabboubi R, Souissi N (2020) Effect of melatonin on inflammatory response to prolonged exercise. Biol Rhythm Res 51:560-565

122. Leonardo-Mendonça RC, Ocaña-Wilhelmi J, de Haro T, de Teresa-Galván C, Guerra-Hernández E, Rusanova I, Fernández-Ortiz M, Sayed RKA, Escames G, Acuña-Castroviejo D (2017) The benefit of a supplement with the antioxidant melatonin on redox status and muscle damage in resistance-trained athletes. Appl Physiol Nutr Metab 42(7):700-707

123. Czuczejko J, Sielski Ł, Woźniak B, Woźniak A, Szewczyk-Golec K (2019) Melatonin supplementation improves oxidative and inflammatory state in the blood of professional athletes during the preparatory period for competitions. Free Radic Res 53(2):198-209

124. Cheikh M, Makhlouf K, Ghattassi K, Graja A, Ferchichi S, Kallel C, Houda M, Souissi N, Hammouda O (2020) Melatonin ingestion after exhaustive late-evening exercise attenuate muscle damage, oxidative stress, and inflammation during intense short term effort in the following day in teenage athletes. Chronobiol Int 37(2):236-247

125. Farjallah MA, Ghattassi K, Graja MLB, A, Boudaya M, Elleuch H, Jammoussi K, Sahnoun Z, Souissi N, Chtourou H, Hammouda O, (2020) Effect of nocturnal melatonin intake on cellular damage and recovery from repeated sprint performance during an intensive training schedule. Chronobiol Int 37(5):686-698

126. Bloomer RJ, Smith WA (2009) Oxidative stress in response to aerobic and anaerobic power testing: influence of exercise training and carnitine supplementation. Res Sports Med 17(1):1-16

127. Kim H, Kim D (2014) Effect of different exercise intensity on blood melatonin density in sleep disordered rats. J Korean Soc Phys Med 9(1):45-53

128. López-Flores M, Luque-Nieto R, Moreira OC, Suarez-Iglesias D, VillaVicente JG (2018) Effects of melatonin on sports performance: a systematic review. J Exerc Physiol Online 21(5):121-138

129. Merry TL, Ristow M (2016) Do antioxidant supplements interfere with skeletal muscle adaptation to exercise training? J Physiol 594(18):5135-5147

130. Kładna A, Aboul-Enein HY, Kruk I (2003) Enhancing effect of melatonin on chemiluminescence accompanying decomposition of hydrogen peroxide in the presence of copper. Free Radic Biol Med 34(12):1544-1554

131. Hong Y, Kim H, Lee S, Jin Y, Choi J, Lee SR, Chang KT, Hong Y (2017) Role of melatonin combined with exercise as a switch-like regulator for circadian behavior in advanced osteoarthritic knee. Oncotarget 8(57):97633-97647

132. Gedikli S, Gelen V, Sengul E, Ozkanlar S, Gur C, Agırbas O, Cakmak F, Kara A (2015) Therapeutic effects of melatonin on liver and kidney damages in intensive exercise model of rats. Endocr Metab Immune Disord Drug Targets 15(4):308-314

133. Martel J, Ojcius DM, Ko YF (2019) Hormetic effects of phytochemicals on health and longevity. Trends Endocrinol Metab 30(6):335-346

134. Kansanen E, Kuosmanen SM, Leinonen H, Levonen AL (2013) The Keap1-Nrf2 pathway: mechanisms of activation and dysregulation in cancer. Redox Biol 1(1):45-49

135. Goldfarb AH, McKenzie MJ, Bloomer RJ (2007) Gender comparisons of exercise-induced oxidative stress: influence of antioxidant supplementation. Appl Physiol Nutr Metab 32(6):1124-1131

\section{Publisher's Note}

Springer Nature remains neutral with regard to jurisdictional claims in published maps and institutional affiliations. 